\title{
TITLE:
}

\section{Analysis and Synthesis of Interconnected Positive Systems}

$\operatorname{AUTHOR}(S)$ :

Ebihara, Yoshio; Peaucelle, Dimitri; Arzelier, Denis

\section{CITATION:}

Ebihara, Yoshio ...[et al]. Analysis and Synthesis of Interconnected

Positive Systems. IEEE Transactions on Automatic Control 2017, 62(2): 652-667

\section{ISSUE DATE:}

2017-02

URL:

http://hdl.handle.net/2433/243178

\section{RIGHT:}

(C) 2016 IEEE. Personal use of this material is permitted. Permission from IEEE must be obtained for all other uses, in any current or future media, including reprinting/republishing this material for advertising or promotional purposes, creating new collective works, for resale or redistribution to servers or lists, or reuse of any copyrighted component of this work in other works.; この論文は出版社版でありません。引用の際には出版社版をご確認ご利用ください。;This is not the published version. Please cite only the published version. 


\title{
Analysis and Synthesis of Interconnected Positive Systems
}

\author{
Yoshio Ebihara, Dimitri Peaucelle, and Denis Arzelier
}

\begin{abstract}
This paper is concerned with the analysis and synthesis of interconnected systems constructed from heterogeneous positive subsystems and a nonnegative interconnection matrix. We first show that admissibility, to be defined in this paper, is an essential requirement in constructing such interconnected systems. Then, we clarify that the interconnected system is admissible and stable if and only if a Metzler matrix, which is built from the coefficient matrices of positive subsystems and the nonnegative interconnection matrix, is Hurwitz stable. By means of this key result, we further provide several results that characterize the admissibility and stability of the interconnected system in terms of the Frobenius eigenvalue of the interconnection matrix and the weighted $L_{1}$-induced norm of the positive subsystems again to be defined in this paper. Moreover, in the case where every subsystem is SISO, we provide explicit conditions under which the interconnected system has the property of persistence, i.e., its state converges to a unique strictly positive vector (that is known in advance up to a strictly positive constant multiplicative factor) for any nonnegative and nonzero initial state. As an important consequence of this property, we show that the output of the interconnected system converges to a scalar multiple of the right eigenvector of a nonnegative matrix associated with its Frobenius eigenvalue, where the nonnegative matrix is nothing but the interconnection matrix scaled by the steady-stage gains of the positive subsystems. This result is then naturally and effectively applied to formation control of multi-agent systems with positive dynamics. This result can be seen as a generalization of a well-known consensus algorithm that has been basically applied to interconnected systems constructed from integrators.
\end{abstract}

Index Terms-positive systems, interconnection, admissibility, stability, multi-agent systems, formation control.

\section{INTRODUCTION}

Recently, systems of interest in the field of engineering, biology, economics, etc., have become more complex and largerscaled, and as such intensive research effort has been made for developing dedicated analysis and synthesis tools. The issue is how to derive sharpened analysis and synthesis conditions exploiting the properties of subsystems and interconnection structure [18], [28], [27], [13]. In this paper, we are particularly interested in the case where the subsystems are positive. A dynamical system is said to be (internally) positive if its state and output are nonnegative for any nonnegative initial state and nonnegative input [12], [21]. This property arises naturally in biology, network communications, economics, and probabilistic systems. Moreover, simple dynamical systems

Y. Ebihara is with the Department of Electrical Engineering, Kyoto University, Kyotodaigaku-Katsura, Nishikyo-ku, Kyoto 615-8510, Japan, he was also with CNRS, LAAS, 7 avenue du Colonel Roche, F-31400 Toulouse, France, in 2011. D. Peaucelle and D. Arzelier are with CNRS, LAAS, 7 avenue du Colonel Roche, F-31400 Toulouse, France, they are also with Univ. de Toulouse, LAAS, F-31400 Toulouse, France.

Corresponding author: Y. Ebihara, Tel/Fax: +81-75-383-2252, e-mail: ebihara@kuee.kyoto-u.ac.jp. such as integrators and first-order lags and their series/parallel connections are all positive, and these are often employed as typical models of moving objects. Even though their dynamics are very simple, the behavior of interconnected systems constructed from them is complicated and deserves investigation especially in the study area of multi-agent systems [14], [28], [27], [38], [39]. This fact naturally leads us to focus on interconnected systems constructed from positive subsystems.

The interconnected system of interest in this paper is constructed from heterogeneous positive subsystems and a nonnegative interconnection matrix. In the past, interconnected systems with nonnegative interconnection matrices (or more precisely, interconnection matrices with nonnegative offdiagonal elements) are studied extensively, see, ex., [41], [42]. A typical example is the case where the interconnection matrix is given based on the graph-Laplacian. In addition, subsystems of interest are often linear and positive as in the case where they are simple integrators [28], [27]. Nowadays positive system theory is fully matured and remarkable results have been obtained by making full use of the positivity [3], [35], [16], [1], [31], [32], [37], [25], [5], [40]. However, the positivity of subsystems and the nonnegativity of interconnection matrices have never been used actively to obtain sharpened analysis and synthesis results under the interconnected systems settings. Our goal in this paper is then to provide such sharpened results and build a solid theoretical basis for the treatment of interconnected systems constructed from positive subsystems and a nonnegative interconnection matrix.

As the first and an important contribution, we show that admissibility, to be defined in this paper, is an essential requirement in constructing interconnected systems of interest. The admissibility is seemingly a sufficient condition for the well-posedness and the positivity of the interconnected system. However, it has deeper implication, and we clarify that the interconnected system without admissibility is of no use in practice since it is fragile against communication delays. On the basis of this preliminary result, we next clarify that the interconnected system is admissible and stable if and only if a Metzler matrix, which is built from the coefficient matrices of the positive subsystems and the interconnection matrix, is Hurwitz stable. By means of this key result, we further provide several results that characterize the admissibility and stability of the interconnected systems in terms of the Frobenius eigenvalue of the interconnection matrix and the weighted $L_{1}$ induced norm of positive subsystems again to be defined in this paper. Moreover, in the case where every subsystem is SISO, we provide explicit conditions under which the interconnected system has the property of persistence, i.e., its state converges to a unique strictly positive vector (that is known in advance 
up to a strictly positive constant multiplicative factor) for any nonnegative and nonzero initial state. As an important consequence of this property, we show that the output of the interconnected system converges to a scalar multiple of the right eigenvector of a nonnegative matrix associated with its Frobenius eigenvalue, where the nonnegative matrix is nothing but the interconnection matrix scaled by the steadystage gains of subsystems. This result is then naturally and effectively applied to formation control of multi-agent systems [14], [28], [27], [38], [39]. For multiple agents that move over a plane, the goal is to design a communication scheme over the agents with respect to each agent's position so that prescribed formation can be achieved. We show that such communication scheme synthesis is possible even if the agents have different dynamics (and hence heterogeneous) as long as they are positive and stable. Moreover, the synthesis condition is given by linear equation that depends only on the steady-state gains of subsystems. We derive this sharpened result by making full use of the positivity. The literature on formation control is quite extensive, and we have not attempted a thorough review of the control scheme proposed here. However, as illustrated later, the current result can be seen as a generalization of a well-known consensus algorithm that has been basically applied to interconnected systems constructed from integrators [27]. In addition, we emphasize that our results essentially concern consensus-based output control of interconnected heterogeneous positive systems, and this is in stark contrast with recent results [40] on state consensus of interconnected homogeneous positive systems where homogeneousness drastically facilitates the treatment.

We finally note that this paper gathers the results in [8], [10], [9] with explicit proofs for technical lemmas and theorems. Moreover, we extend the persistence related results in [10], [9] to the case where the steady-state gains of subsystems are not uniform, and to the case where the interconnection matrix is not irreducible (i.e., reducible), and also apply these latest results to an energy management problem in DC-grids.

We use the following notations. For given two matrices $A$ and $B$ of the same size, we write $A>B(A \geq B)$ if $A_{i j}>$ $B_{i j}\left(A_{i j} \geq B_{i j}\right)$ holds for all $(i, j)$, where $A_{i j}$ stands for the $(i, j)$-entry of $A$. In relation to this notation, we also define $\mathbb{R}_{++}^{n}:=\left\{x \in \mathbb{R}^{n}: x>0\right\}$ and $\mathbb{R}_{+}^{n}:=\left\{x \in \mathbb{R}^{n}: x \geq 0\right\}$. We also define $\mathbb{R}_{++}^{n \times m}$ and $\mathbb{R}_{+}^{n \times m}$ with obvious modifications. In addition, we denote by $\mathbb{D}_{++}^{n \times n}$ the set of diagonal matrices of size $n$ with all diagonal elements being strictly positive. For $A \in \mathbb{R}^{n \times n}$, we denote by $\sigma(A)$ and $\rho(A)$ the set of the eigenvalues of $A$ and the spectral radius of $A$, respectively. For $A \in \mathbb{R}_{+}^{n \times n}$, Theorem 8.3.1 in [19] states that there is an eigenvalue equal to $\rho(A)$. This eigenvalue is often called the Frobenius eigenvalue and denoted by $\lambda_{\mathrm{F}}(A)$ in this paper. For a given vector $x \in \mathbb{R}^{n}$, we define its 1 -norm by $\|x\|_{1}:=$ $\sum_{i=1}^{n}\left|x_{i}\right|$. In addition, for $s(t): \mathbb{R}_{+} \rightarrow \mathbb{R}^{n}$, we define its $L_{1}$-norm by $\|s\|_{1}:=\int_{0}^{\infty}\|s(t)\|_{1} d t$. Finally, we define the families of functions $L_{1}^{n}, L_{1+}^{n}$ as follows:

$$
\begin{aligned}
& L_{1}^{n}:=\left\{s \mid s(t): \mathbb{R}_{+} \rightarrow \mathbb{R}^{n},\|s\|_{1}<\infty\right\} \\
& L_{1+}^{n}:=\left\{s \mid s(t): \mathbb{R}_{+} \rightarrow \mathbb{R}_{+}^{n},\|s\|_{1}<\infty\right\}
\end{aligned}
$$

\section{PRELIMINARIES}

In this section, we gather basic definitions and fundamental results for positive systems.

Definition 1 (Metzler Matrix): [12] A matrix $A \in \mathbb{R}^{n \times n}$ is said to be Metzler if its off-diagonal entries are all nonnegative, i.e., $A_{i j} \geq 0(i \neq j)$.

In the following, we denote by $\mathbb{M}^{n \times n}\left(\mathbb{H}^{n \times n}\right)$ the set of the Metzler (Hurwitz stable) matrices of size $n$. Under these notations, the next lemmas hold.

Lemma 1: [12], [21], [26] For a given $A \in \mathbb{M}^{n \times n}$, the following conditions are equivalent.

(i) The matrix $A$ is Hurwitz stable, i.e., $A \in \mathbb{H}^{n \times n}$.

(ii) The matrix $A$ is nonsingular and $A^{-1} \leq 0$.

(iii) There exists $h \in \mathbb{R}_{++}^{n}$ such that $h^{T} A<0$.

(iv) For any $g \in \mathbb{R}_{+}^{n} \backslash\{0\}$, the vector $A g$ has at least one strictly negative entry.

Lemma 2: For given $P \in \mathbb{M}^{n_{1} \times n_{1}}, Q \in \mathbb{R}_{+}^{n_{1} \times n_{2}}, R \in$ $\mathbb{R}_{+}^{n_{2} \times n_{1}}$, and $S \in \mathbb{M}^{n_{2} \times n_{2}}$, the following conditions are equivalent.

(i) $\Pi:=\left[\begin{array}{ll}P & Q \\ R & S\end{array}\right] \in \mathbb{H}^{\left(n_{1}+n_{2}\right) \times\left(n_{1}+n_{2}\right)}$.

(ii) $P \in \mathbb{H}^{n_{1} \times n_{1}}, S-R P^{-1} Q \in \mathbb{H}^{n_{2} \times n_{2}}$.

(iii) $S \in \mathbb{H}^{n_{2} \times n_{2}}, P-Q S^{-1} R \in \mathbb{H}^{n_{1} \times n_{1}}$.

The proof of Lemma 2 is given in the appendix section, Subsection IX-A.

To move on to the definition of positive systems, consider the linear system $G$ described by

$$
G:\left\{\begin{array}{l}
\dot{x}=A x+B w \\
z=C x+D w
\end{array}\right.
$$

where $A \in \mathbb{R}^{n \times n}, B \in \mathbb{R}^{n \times n_{w}}, C \in \mathbb{R}^{n_{z} \times n}$, and $D \in$ $\mathbb{R}^{n_{z} \times n_{w}}$. The definition of positive systems and a related basic result are given in the following.

Definition 2 (Positive Linear System): [12] The linear system (1) is said to be positive if its state and output are both nonnegative for any nonnegative initial state and nonnegative input.

Remark 1: In the literature, a system satisfying the condition in Definition 2 is often called internally positive, to make a clear distinction from externally positive systems. Since we only deal with internally positive systems in this paper, we simply denote it by positive as in Definition 2 .

Proposition 1: [12] The system (1) is positive if and only if $A \in \mathbb{M}^{n \times n}, B \in \mathbb{R}_{+}^{n \times n_{w}}, C \in \mathbb{R}_{+}^{n_{z} \times n}$, and $D \in \mathbb{R}_{+}^{n_{z} \times n_{w}}$.

We next introduce the weighted $L_{1}$-induced norm of positive systems. It turns out in the next section that the weighted $L_{1}$-induced norm plays an important role in characterizing the stability of interconnected positive systems.

Definition 3: Suppose $G$ given by (1) is positive and $x(0)=$ 0 . Then, its weighted $L_{1}$-induced norm associated with weighting vectors $q_{z} \in \mathbb{R}_{++}^{n_{z}}$ and $q_{w} \in \mathbb{R}_{++}^{n_{w}}$ is defined by

$$
\left\|G_{q_{z}, q_{w}}\right\|_{1+}:=\sup _{\left\|q_{w}^{T} w\right\|_{1}=1, w \in L_{1+}^{n_{w}}}\left\|q_{z}^{T} z\right\|_{1} \text {. }
$$

Remark 2: The standard $L_{1}$-induced norm of $G$ given by (1) is defined as follows [15]:

$$
\|G\|_{1}:=\sup _{\|w\|_{1}=1, w \in L_{1}^{n_{w}}}\|z\|_{1} .
$$


From the positivity of $G$, we can easily confirm that the two $L_{1}$-induced norms given above can be linked by

$$
\left\|G_{q_{z}, q_{w}}\right\|_{1+}=\left\|Q_{z} G Q_{w \mathbf{1}^{n_{z}, \mathbf{1}^{n_{w}}}}^{-1}\right\|_{1+}=\left\|Q_{z} G Q_{w}^{-1}\right\|_{1},
$$

where $\quad Q_{z} \quad:=\operatorname{diag}\left(q_{z, 1}, \cdots, q_{z, n_{z}}\right), \quad Q_{w} \quad:=$ $\operatorname{diag}\left(q_{w, 1}, \cdots, q_{w, n_{w}}\right)$ and $\mathbf{1}^{n_{z}}$ stands for the all-ones vector of size $n_{z}$. The state space matrices of $Q_{z} G Q_{w}^{-1}$ are given by $\left(A, B Q_{w}^{-1}, Q_{z} C, Q_{z} D Q_{w}^{-1}\right)$. Namely, as the denomination "weighted" $L_{1}$-induced norm stands, $\left\|G_{q_{z}, q_{w}}\right\|_{1+}$ coincides with the standard $L_{1}$-induced norm with weightings (or scalings) on the input and output signals. The vector representation of weightings as in $q_{z}$ and $q_{w}$ rather than the matrix representation as in $Q_{z}$ and $Q_{w}$ is useful in characterizing the weighted $L_{1}$-induced norm and the stability of interconnected positive systems by linear inequalities. This is illustrated in the next theorem.

Theorem 1: Suppose $G$ given by (1) is positive. Then, for given $q_{z} \in \mathbb{R}_{++}^{n_{z}}, q_{w} \in \mathbb{R}_{++}^{n_{w}}$, and $\gamma>0$, the following conditions are equivalent.

(i) The matrix $A \in \mathbb{M}^{n \times n}$ is Hurwitz stable and $\left\|G_{q_{z}, q_{w}}\right\|_{1+}<\gamma$.

(ii) There exists $h \in \mathbb{R}_{++}^{n}$ such that

$$
\left[h^{T} A+q_{z}^{T} C \stackrel{h^{T}}{\left.B+q_{z}^{T} D-\gamma q_{w}^{T}\right]<0 .}\right.
$$

(iii) The matrix $A \in \mathbb{M}^{n \times n}$ is Hurwitz stable and the following inequality holds:

$$
q_{z}^{T} G(0)<\gamma q_{w}^{T} \text {. }
$$

Here, $G(s)$ is the transfer matrix of the system $G$ defined by $G(s):=C(s I-A)^{-1} B+D$.

The inequality (5) in (ii) is linear with respect to the decision variable $h \in \mathbb{R}_{++}^{n}$. From this linear inequality, we see that the weighted $L_{1}$-induced norm $\left\|G_{q_{z}, q_{w}}\right\|_{1+}$ can be computed by solving a linear programming problem (LP) given as follows: $\inf _{\gamma, h \in \mathbb{R}_{++}^{n}} \quad \gamma$ subject to (5). The condition (6) in (iii) is more compact and characterizes the weighted $L_{1}$-induced norm in a closed form (see Corollary 1 below as well).

If we let $q_{z}=\mathbf{1}^{n_{z}}$ and $q_{w}=\mathbf{1}^{n_{w}}$, the definition (2) essentially reduces to the standard $L_{1}$-induced norm as we noted in (4). This standard $L_{1}$-induced norm is employed as a performance index in recent studies on switched positive systems [43], [44]. Moreover, this standard $L_{1}$-induced norm is used in [5] as a useful tool for robust stability analysis of uncertain positive systems.

Even though related discussions on the proof of Theorem 1 can be found, for example, in [31], [5], we give a detailed proof of Theorem 1 in the appendix section, Subsection IX-B, for completeness. The next corollary directly follows from (iii) in Theorem 1.

Corollary 1: Suppose $G$ given by (1) is positive and stable. Then, for given $q_{z} \in \mathbb{R}_{++}^{n_{z}}, q_{w} \in \mathbb{R}_{++}^{n_{w}}$, the weighted $L_{1^{-}}$ induced norm $\left\|G_{q_{z}, q_{w}}\right\|_{1+}$ is given by

$$
\left\|G_{q_{z}, q_{w}}\right\|_{1+}=\min \gamma \text { subject to } q_{z}^{T} G(0) \leq \gamma q_{w}^{T}
$$

or equivalently,

$$
\left\|G_{q_{z}, q_{w}}\right\|_{1+}=\max _{i} \frac{\left(q_{z}^{T} G(0)\right)_{i}}{q_{w, i}} .
$$

This corollary implies that, if $G$ given by (1) is stable and SISO, we have $\left\|G_{1,1}\right\|_{1+}=G(0)$. Namely, the unweighted $L_{1}$-induced norm coincides with the steady-state gain.

\section{STABility ANALYSis OF \\ INTERCONNECTED Positive Systems}

\section{A. Interconnected Positive Systems and Admissibility}

Let us consider the positive subsystem $G_{i}(i=1, \cdots, N)$ represented by

$$
\begin{aligned}
& G_{i}:\left\{\begin{array}{l}
\dot{x}_{i}=A_{i} x_{i}+B_{i} w_{i}, \\
z_{i}=C_{i} x_{i}+D_{i} w_{i},
\end{array}\right. \\
& A_{i} \in\left\{\mathbb{M}^{n_{i} \times n_{i}} \cap \mathbb{H}^{n_{i} \times n_{i}}\right\}, B_{i} \in \mathbb{R}_{+}^{n_{i} \times n_{w_{i}}}, \\
& C_{i} \in \mathbb{R}_{+}^{n_{z_{i}} \times n_{i}}, D_{i} \in \mathbb{R}_{+}^{n_{z_{i}} \times n_{w_{i}}} .
\end{aligned}
$$

As clearly shown in (9), we have assumed that $G_{i}(i=$ $1, \cdots, N)$ are all stable.

With these positive and stable subsystems, let us define a positive and stable system $\mathcal{G}$ by $\mathcal{G}:=\operatorname{diag}\left(G_{1}, \cdots, G_{N}\right)$. The state space realization of $\mathcal{G}$ is given by

$$
\mathcal{G}:\left\{\begin{aligned}
\dot{\hat{x}} & =\mathcal{A} \widehat{x}+\mathcal{B} \widehat{w} \\
\widehat{z} & =\mathcal{C} \widehat{x}+\mathcal{D} \widehat{w}
\end{aligned}\right.
$$

where

$$
\begin{aligned}
& \mathcal{A}:=\operatorname{diag}\left(A_{1}, \cdots, A_{N}\right), \mathcal{B}:=\operatorname{diag}\left(B_{1}, \cdots, B_{N}\right), \\
& \mathcal{C}:=\operatorname{diag}\left(C_{1}, \cdots, C_{N}\right), \mathcal{D}:=\operatorname{diag}\left(D_{1}, \cdots, D_{N}\right), \\
& \widehat{x}:=\left[\begin{array}{c}
x_{1} \\
\vdots \\
x_{N}
\end{array}\right] \in \mathbb{R}^{n_{\widehat{x}}}, \widehat{w}:=\left[\begin{array}{c}
w_{1} \\
\vdots \\
w_{N}
\end{array}\right] \in \mathbb{R}^{n_{\widehat{w}}}, \widehat{z}:=\left[\begin{array}{c}
z_{1} \\
\vdots \\
z_{N}
\end{array}\right] \in \mathbb{R}^{n_{\widehat{z}}}, \\
& n_{\widehat{x}}:=\sum_{i=1}^{N} n_{i}, \quad n_{\widehat{w}}:=\sum_{i=1}^{N} n_{w_{i}}, \quad n_{\widehat{z}}:=\sum_{i=1}^{N} n_{z_{i}} .
\end{aligned}
$$

For a given interconnection matrix $\Omega \in \mathbb{R}_{+}^{n_{\hat{w}} \times n_{\widehat{z}}}$, we are interested in the stability and the performance of the interconnected system $\mathcal{G} \star \Omega$ defined by (10) and $\widehat{w}=\Omega \widehat{z}$. In relation to the well-posedness of this interconnection, we make the next definition.

Definition 4: The interconnected system $\mathcal{G} \star \Omega$ is said to be admissible if the Metzler matrix $\mathcal{D} \Omega-I$ is Hurwitz stable.

In the following, we require the admissibility of the interconnected system $\mathcal{G} \star \Omega$ whenever we analyze its stability and performance. The meaning of this presupposition, and its rationality as well, can be explained as follows.

(i) If $\operatorname{det}(\mathcal{D} \Omega-I) \neq 0$, then the interconnection is well-posed, and the state-space description of the interconnected system is represented by

$$
\dot{\widehat{x}}=\mathcal{A}_{\mathrm{cl}} \widehat{x}, \quad \mathcal{A}_{\mathrm{cl}}:=\mathcal{A}+\mathcal{B} \Omega(I-\mathcal{D} \Omega)^{-1} \mathcal{C} .
$$

(ii) The Metzler matrix $\mathcal{D} \Omega-I$ is Hurwitz and hence $(I-\mathcal{D} \Omega)^{-1} \geq 0$ holds from (ii) of Lemma 1 . Therefore the matrix $A_{\mathrm{cl}}$ given above is Metzler. It follows that the positive nature of the subsystems $G_{i}(i=1, \cdots, N)$ is inherited to the interconnected system, i.e., the nonnegativity of the states $x_{i}(i=1, \cdots, N)$ for any nonnegative initial states is still preserved under the interconnection.

(iii) More strongly, we can say that the admissibility for the interconnected positive system $\mathcal{G} \star \Omega$ is mandatory from a control engineering point of view. This is because $\mathcal{G} \star \Omega$ that does not satisfy the admissibility is of no use in practice since it is fragile against communication delays. To be more precise, let us consider the case where $\mathcal{G} \star \Omega$ is perturbed as $\mathcal{G} \star\left(\Omega e^{-s h}\right)$, 
where $h>0$ is a uniform delay on communication over subsystems. Then, if $\mathcal{G} \star \Omega$ does not satisfy the admissibility, we see that $\mathcal{G} \star\left(\Omega e^{-s h}\right)$ is unstable for any $h>0$. Indeed, if $\mathcal{G} \star \Omega$ is not admissible, then the matrix $\mathcal{D} \Omega-I$ is not Hurwitz stable by definition and hence $\rho(\mathcal{D} \Omega) \geq 1$ holds. From this fact and [23], we see that the spectral radius of a monodromy operator associated with $\mathcal{G} \star\left(\Omega e^{-s h}\right)$ is not less than one and hence $\mathcal{G} \star\left(\Omega e^{-s h}\right)$ is unstable for any $h>0$. Therefore, even if we build theoretical results for interconnected systems that do not satisfy the admissibility, such results are of no use since communication delays are unavoidable in practice (even if they are very small).

We also note that the admissibility is no more an issue if $\mathcal{D}=0$, since in this case we have $\mathcal{A}_{\mathrm{cl}}=\mathcal{A}+\mathcal{B} \Omega \mathcal{C} \in \mathbb{M}^{n_{\widehat{x}} \times n_{\widehat{x}}}$ and hence $\mathcal{G} \star \Omega$ is always well-posed, positive, and its stability is preserved against arbitrary (time-invariant) communication delays [17].

For the admissibility and stability of the interconnected system $\mathcal{G} \star \Omega$, we can obtain the next lemma that plays an important role in this paper.

Lemma 3: The interconnected system $\mathcal{G} \star \Omega$ is admissible and stable if and only if the Metzler matrix

$$
\Pi:=\left[\begin{array}{cc}
\mathcal{A} & \mathcal{B} \Omega \\
\mathcal{C} & \mathcal{D} \Omega-I
\end{array}\right]
$$

is Hurwitz stable.

Proof of Lemma 3: From Definition 4, the interconnected system $\mathcal{G} \star \Omega$ is admissible and stable if and only if the Metzler matrices $\mathcal{D} \Omega-I$ and $\mathcal{A}_{\mathrm{cl}}=\mathcal{A}+\mathcal{B} \Omega(I-\mathcal{D} \Omega)^{-1} \mathcal{C}$ are both Hurwitz stable. Thus the assertion readily follows from Lemma 2.

From this key lemma, we can obtain various conditions for the admissibility and stability of the interconnected system according to the properties of the subsystems $G_{i}(i=1, \cdots, N)$ and the interconnection matrix $\Omega$. Typical examples are given in the following two subsections.

\section{B. Stability for General Interconnection Structure}

The first result concerns the interconnected system shown in Fig. 1 for the case $N=3$. The interconnection shown in Fig. 1 is general, in the sense that (i) every subsystem provides different output signals to the rest of the subsystems, (ii) every subsystem receives input signals from the rest of the subsystems independently, and (iii) there is no restriction on the size of input/output signals. For the admissibility and stability of the interconnected system, we can obtain the next theorem. Note that this theorem also includes the state-space description of subsystems allowing the assumed interconnection structure.

Theorem 2: Let us consider the case where the $i$-th stable subsystem $G_{i}$ represented by (9) has the following specific structure:

$$
\begin{aligned}
& G_{i}:\left\{\begin{array}{l}
\dot{x}_{i}=A_{i} x_{i}+\sum_{k=1, k \neq i}^{N} B_{i k} w_{i k}, \\
z_{j i}=C_{j i} x_{i}+\sum_{k=1, k \neq i}^{N} D_{j i k} w_{i k} \quad(j \neq i),
\end{array}\right. \\
& A_{i} \in\left\{\mathbb{M}^{n_{i} \times n_{i}} \cap \mathbb{H}^{n_{i} \times n_{i}}\right\}, B_{i k} \in \mathbb{R}_{+}^{n_{i} \times n_{w_{i k}}},
\end{aligned}
$$

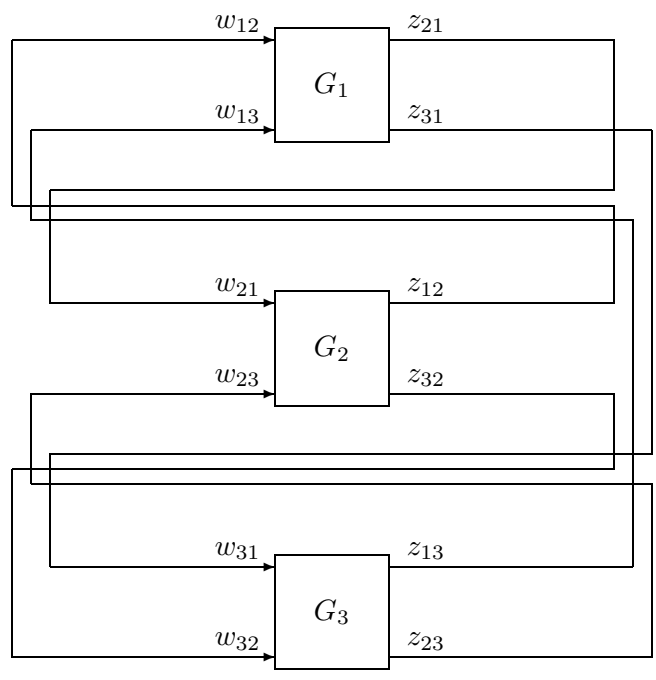

Fig. 1. Interconnected positive system $(N=3)$.

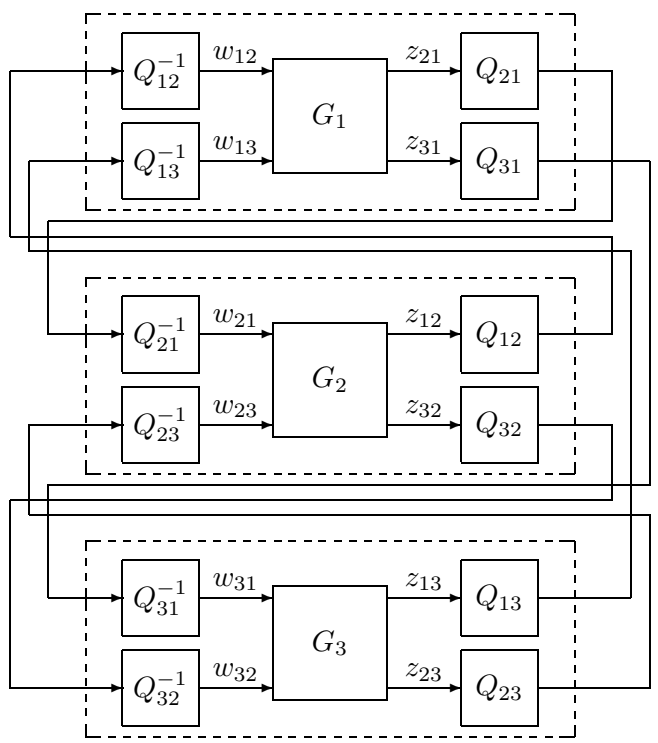

Fig. 2. Weightings on input and output signals.

$$
C_{j i} \in \mathbb{R}_{+}^{n_{z_{j i}} \times n_{i}}, D_{j i k} \in \mathbb{R}_{+}^{n_{z_{j i}} \times n_{w_{i k}}} .
$$

We assume that the size of $w_{i j}$ and $z_{i j}$ are identical, and $N$ subsystems are interconnected by

$$
w_{i j}=z_{i j}(i, j=1, \cdots, N, i \neq j) \text {. }
$$

Then, the interconnected system is admissible and stable if and only if there exist weighting vectors $q_{i j} \in \mathbb{R}_{++}^{n_{w_{i j}}}(i, j=$ $1, \cdots, N, i \neq j$ ) such that

$$
\begin{aligned}
& \left\|G_{i, q_{z, i}, q_{w, i}}\right\|_{1+}<1 \text {, } \\
& q_{z, i}=\left[\begin{array}{llllll}
q_{1, i}^{T} & \cdots & q_{i-1, i}^{T} & q_{i+1, i}^{T} & \cdots & q_{N, i}^{T}
\end{array}\right]^{T}, \\
& q_{w, i}=\left[\begin{array}{llllll}
q_{i, 1}^{T} & \cdots & q_{i, i-1}^{T} & q_{i, i+1}^{T} & \cdots & q_{i, N}^{T}
\end{array}\right]^{T} \\
& (i=1, \cdots, N) .
\end{aligned}
$$

Remark 3: From (5) of Theorem 1, we see that the inequality condition (17) in Theorem 2 is linear with respect to the weighting vectors $q_{i j} \in \mathbb{R}_{++}^{n_{w_{i j}}}(i, j=1, \cdots, N, i \neq j)$ and hence easily verifiable.

As noted, the interconnection structure assumed in Theorem 2 is illustrated in Fig. 1 for the case $N=3$. The subscripts $(i, j)$ of $w_{i j}$ and $z_{i j}$ indicate that these are the signals that flow from the subsystem $j$ to the subsystem $i$. By defining 


$$
\begin{aligned}
z_{i}= & {\left[\begin{array}{llllll}
z_{1, i}^{T} & \cdots & z_{i-1, i}^{T} & z_{i+1, i}^{T} & \cdots & z_{N, i}^{T}
\end{array}\right]^{T} } \\
w_{i}= & {\left[\begin{array}{llllll}
w_{i, 1}^{T} & \cdots & w_{i, i-1}^{T} & w_{i, i+1}^{T} & \cdots & w_{i, N}^{T}
\end{array}\right]^{T} } \\
&
\end{aligned}
$$

and by representing the interconnection (16) by $\widehat{w}=\Omega \widehat{z}$, we can see that the interconnected system can be represented by $\mathcal{G} \star \Omega$. For $N=3$, the interconnection matrix is given by

$$
\Omega=\left[\begin{array}{cc:cc:cc}
0 & 0 & I_{n_{w_{12}}} & 0 & 0 & 0 \\
0 & 0 & 0 & 0 & I_{n_{w_{13}}} & 0 \\
\hdashline I_{n_{w_{21}}} & 0 & 0 & 0 & 0 & 0 \\
0 & 0 & 0 & 0 & 0 & I_{n_{w_{23}}} \\
\hdashline 0 & I_{n_{w_{31}}} & 0 & 0 & 0 & 0 \\
0 & 0 & 0 & I_{n_{w_{32}}} & 0 & 0
\end{array}\right] .
$$

By applying Lemma 3 to the resulting interconnected system $\mathcal{G} \star \Omega$, we obtain Theorem 2 . The complete proof is given in the appendix section, Subsection IX-C.

The implication of the theorem is that the interconnected system $\mathcal{G} \star \Omega$ is admissible and stable if and only if there exists a set of weighting vectors that renders the weighted $L_{1}$-induced norm of each positive subsystem less than unity. Namely, the condition for the admissibility and stability is separated into the $L_{1}$-induced norm conditions of subsystems. In this sense, the weighting vectors work as separators that have played important roles for stability analysis of general linear systems [20], [34], [29]. Another interpretation is that, as we usually do for separators as well, the weighting vectors serve as scalings for input and output signals. Indeed, from the link (4), we see that $\mathcal{G} \star \Omega$ is admissible and stable if and only if the standard $L_{1}$-induced norms of scaled systems (i.e., the systems encircled by dashed lines in Fig. 2) are less than unity, where $Q_{i j}=\operatorname{diag}\left(q_{i j}\right)$. What is interesting here is that such scaling-based stability condition is necessary and sufficient, which is hardly achievable for interconnected systems constructed from general (non-positive) linear systems.

\section{Stability for Interconnection with SISO Positive Subsystems}

The results in Theorem 2 are valid for MIMO positive subsystems. On the other hand, in the case where every subsystem is SISO, conditions for the admissibility and stability for the interconnected system $\mathcal{G} \star \Omega$ can be drastically simplified as we see in the next two theorems.

Theorem 3: Let us consider the case where the stable subsystems $G_{i}(i=1, \cdots, N)$ represented by (9) are all SISO. Then, for a given $\Omega \in \mathbb{R}_{+}^{N \times N}$, the interconnected system $\mathcal{G} \star \Omega$ is admissible and stable if and only if $\lambda_{\mathrm{F}}(\Psi \Omega)<1$ where $\Psi \in \mathbb{R}_{+}^{N \times N}$ is constructed from the unweighted $L_{1}$-induced norm (i.e., the steady-state gain) of each subsystem as in $\Psi:=\operatorname{diag}\left(\left\|G_{1,1,1}\right\|_{1+}, \cdots,\left\|G_{N, 1,1}\right\|_{1+}\right)=$ $\operatorname{diag}\left(G_{1}(0), \cdots, G_{N}(0)\right)$.

Proof of Theorem 3: From Lemma 3, the interconnected system $\mathcal{G} \star \Omega$ is admissible and stable if and only if the Metzler matrix $\Pi$ defined by (14) is Hurwitz stable. From Lemma 2 and the fact that $\left\|G_{i, 1,1}\right\|_{1+}=G_{i}(0)=-C_{i} A_{i}^{-1} B_{i}+D_{i}(i=$ $1, \cdots, N)$, this condition holds if and only if both the Metzler matrices $\mathcal{A}$ and $\mathcal{D} \Omega-I-\mathcal{C} \mathcal{A}^{-1} \mathcal{B} \Omega=\Psi \Omega-I$ are Hurwitz stable. Thus the assertion readily follows since $\mathcal{A}$ is Hurwitz stable from the assumption $A_{i} \in\left\{\mathbb{M}^{n_{i} \times n_{i}} \cap \mathbb{H}^{n_{i} \times n_{i}}\right\}$.
Theorem 4: Let us consider the case where the stable subsystems $G_{i}(i=1, \cdots, N)$ represented by (9) are all SISO and share identical steady-state gain $\gamma>0$, i.e., $G_{1}(0)=$ $\cdots=G_{N}(0)=\gamma$. Then, for a given $\Omega \in \mathbb{R}_{+}^{N \times N}$, the interconnected system $\mathcal{G} \star \Omega$ is admissible and stable if and only if $\gamma \lambda_{\mathrm{F}}(\Omega)<1$.

Proof of Theorem 4: Obvious from Theorem 3.

These three theorems clearly show that the admissibility and stability of interconnected positive systems can be fully characterized in terms of weighted $L_{1}$-induced norms of subsystems and the Frobenius eigenvalue of the interconnection matrix $\Omega$ scaled by $\Psi$. In particular, if all subsystems are SISO, we see from Theorems 3 and 4 that the interconnected system $\mathcal{G} \star \Omega$ is on the stability boundary if $\lambda_{\mathrm{F}}(\Psi \Omega)=1$. This simple condition leads us to the persistence analysis of $\mathcal{G} \star \Omega$ as detailed in the next section.

Remark 4: In [33], [30], stability of interconnected nonlinear systems is investigated. In particular, in [33], each nonlinear subsystem is assumed to satisfy a dissipative integral inputto-state stability estimate. Through a comparison principle, stability of the original interconnected nonlinear system can be analyzed by a comparison system, which is an interconnected positive system since its state is composed of the value of Lyapunov function for each subsystem. Once the positive comparison system is obtained which is still nonlinear, the main focus there is to extend linear case stability results such as the condition (iv) of Lemma 1 to nonlinear positive systems. Even though we deal with interconnected LTI positive systems only in this paper, we have shown that sharp stability conditions are obtained with $L_{1}$-induced norms of subsystems and the Frobenius eigenvalue of the interconnection matrix by making full use of the linearity. On the other hand, the paper [33] implies that the stability conditions in this paper can be applied to interconnected nonlinear systems as long as its comparison system is linear. It is true that this assumption is stringent and holds only for a limited class of interconnected nonlinear systems. Still, constructing a linear comparison system paves the way for applying current results to stability analysis of interconnected nonlinear systems.

\section{Persistence Analysis of INTERCONNECTED POSITIVE SYSTEMS}

In this section, we are interested in the persistence of the interconnected system $\mathcal{G} \star \Omega$. After giving our main results on the persistence of $\mathcal{G} \star \Omega$, we show that the persistence results can be applied to formation control of multi-agent systems with positive dynamics.

\section{A. Persistence Analysis}

We first give the precise definition of what we call persistence.

Definition 5: For given positive and stable subsystems $G_{i}(i=1, \cdots, N)$ represented by (9) and interconnection matrix $\Omega \in \mathbb{R}_{+}^{n_{\widehat{w}} \times n_{\widehat{z}}}$, consider the interconnected system $\mathcal{G} \star \Omega$. Then, the interconnected system $\mathcal{G} \star \Omega$ is said to have the property of persistence if it is admissible and if there exist $\xi_{0}, \xi_{\infty} \in \mathbb{R}_{++}^{n_{\widehat{x}}}$ such that 
$\lim _{t \rightarrow \infty} \widehat{x}(t)=\left(\xi_{0}^{T} \widehat{x}(0)\right) \xi_{\infty}$

for any initial state $\widehat{x}(0) \in \mathbb{R}^{n_{\widehat{x}}}$.

This definition requires that the state $\widehat{x}$ of $\mathcal{G} \star \Omega$ converges to a strictly positive scalar multiple of a strictly positive vector as long as $\widehat{x}(0) \in \mathbb{R}_{+}^{n_{\widehat{x}}} \backslash\{0\}$. Namely, all the states $\widehat{x}_{i}\left(i=1, \cdots, n_{\widehat{x}}\right)$ become strictly positive and hence "excited" eventually. This is the reason why we call the property persistence. It is also clear that persistence requires that the interconnected system $\mathcal{G} \star \Omega$ is on the stability boundary.

To state our main results on the persistence of $\mathcal{G} \star \Omega$, i.e., Theorems 5 and 6 given later, we first need to review the definition and related results on irreducible matrices. Similarly to [40], it turns out that the irreducibility of the interconnection matrix plays a crucial role in achieving persistence.

Definition 6: [Reducible Matrix [19] (p. 360)] A matrix $M \in$ $\mathbb{R}^{n \times n}$ is said to be reducible if either

(a) $n=1$ and $M=0$ or

(b) $n \geq 2$ and there exist a permutation matrix $P \in \mathbb{R}^{n \times n}$ and $r$ with $1 \leq r \leq n-1$ such that

$$
P^{T} M P=\left[\begin{array}{cc}
Q & R \\
0_{n-r, r} & S
\end{array}\right], Q \in \mathbb{R}^{r \times r}, S \in \mathbb{R}^{(n-r) \times(n-r)} .
$$

Definition 7: [Irreducible Matrix [19] (p. 361)] A matrix $M \in \mathbb{R}^{n \times n}$ is said to be irreducible if it is not reducible.

Definition 8: [Directed Graph of Matrices [19] (p. 357)] The directed graph of $M \in \mathbb{R}^{n \times n}$, denoted by $\Gamma(M)$, is the directed graph on $n$ nodes $P_{1}, P_{2}, \cdots, P_{n}$ such that there is a directed arc in $\Gamma(M)$ from $P_{i}$ to $P_{j}$ if and only if $M_{i j} \neq 0$ or equivalently, $\operatorname{In}(M)_{i j} \neq 0$. Here, $\operatorname{In}(M)$ stands for the indicator matrix of $M$.

Definition 9: [Strongly Connected Graph [19] (p. 358)] A directed graph $\Gamma$ is said to be strongly connected if between every pair of distinct nodes $P_{i}, P_{j}$ in $\Gamma$ there is a directed path of finite length that begins at $P_{i}$ and ends at $P_{j}$.

Under these definitions, the next results hold.

Proposition 2: [19] (p. 362) For a given $M \in \mathbb{R}^{n \times n}$, the following conditions are equivalent.

(a) $M$ is irreducible.

(b) $\left(I_{n}+\operatorname{In}(M)\right)^{n-1}>0$.

(c) $\Gamma(M)$ is strongly connected.

Proposition 3: [19] (p. 508) Suppose $M \in \mathbb{R}_{+}^{n \times n}$ is irreducible. Then the following conditions hold.

(i) $\rho(M)>0$ and $\rho(M)$ is an eigenvalue of $M$.

(ii) There is a vector $v \in \mathbb{R}_{++}^{n}$ such that $M v=\rho(M) v$.

(iii) $\rho(M)$ is an algebraically (and hence geometrically) simple eigenvalue of $M$.

The next corollary directly follows from Proposition 3, where (iii) is particularly important.

Corollary 2: Suppose $M \in \mathbb{M}^{n \times n}$ is irreducible. Then the following conditions hold where $\alpha:=\max _{\lambda_{\in \sigma}(M)} \operatorname{Re}(\lambda)$.

(i) $\alpha \in \mathbb{R}$ is an algebraically (and hence geometrically) simple eigenvalue of $M$.

(ii) There is a vector $v \in \mathbb{R}_{++}^{n}$ such that $M v=\alpha v$.

(iii) $\operatorname{Re}(\lambda)<\alpha(\forall \lambda \in \sigma(M) \backslash\{\alpha\})$.

We are now ready to state our main result on the persistence of $\mathcal{G} \star \Omega$ and give its proof.
Theorem 5: Let us consider the case where every stable subsystem $G_{i}$ represented by (9) is SISO. Suppose $G_{i}(i=$ $1, \cdots, N)$ and a given interconnection matrix $\Omega \in \mathbb{R}_{+}^{N \times N}$ satisfy the following conditions.

(i) $\left(A_{i}, B_{i}\right)$ is controllable and $\left(A_{i}, C_{i}\right)$ is observable for all $i=1, \cdots, N$.

(ii) The interconnection matrix $\Omega \in \mathbb{R}_{+}^{N \times N}$ is irreducible, i.e., the directed graph $\Gamma(\Omega)$ is strongly connected.

(iii) $\lambda_{\mathrm{F}}(\Psi \Omega)=1$ holds.

Then, for the interconnected system $\mathcal{G} \star \Omega$, the next results hold.

(I) The interconnected system $\mathcal{G} \star \Omega$ is admissible, i.e., the Metzler matrix $\mathcal{D} \Omega-I$ is Hurwitz stable.

(II) The matrix $\mathcal{A}_{\mathrm{cl}}$ given by (13) satisfies $\sigma\left(\mathcal{A}_{\mathrm{cl}}\right) \subset \overline{\mathbb{C}_{-}}$, i.e., $\operatorname{Re}(\lambda) \leq 0\left(\forall \lambda \in \sigma\left(\mathcal{A}_{\mathrm{cl}}\right)\right)$.

(III) If we denote the right and left eigenvectors of $\Psi \Omega \in \mathbb{R}_{+}^{N \times N}$ associated with its Frobenius eigenvalue $\lambda_{\mathrm{F}}(\Psi \Omega)=1$ by $v_{\mathrm{R}} \in \mathbb{R}_{++}^{N}$ and $v_{\mathrm{L}} \in \mathbb{R}_{++}^{N}$, respectively, we have $\mathcal{A}_{\mathrm{cl}} \xi_{\mathrm{R}}=0$ and $\xi_{\mathrm{L}}^{T} \mathcal{A}_{\mathrm{cl}}=0$ where $\xi_{\mathrm{R}}=-\mathcal{A}^{-1} \mathcal{B} \Psi^{-1} v_{\mathrm{R}} \in \mathbb{R}_{+\widehat{T}_{+}}^{n_{\widehat{C}}}$,

$\xi_{\mathrm{L}}=-\mathcal{A}^{-T} \mathcal{C}^{T} v_{\mathrm{L}} \in \mathbb{R}_{++}^{n_{\widehat{x}}}$, $\xi_{\mathrm{L}}^{T} \xi_{\mathrm{R}}=1$.

Here the eigenvectors $v_{\mathrm{R}}, v_{\mathrm{L}} \in \mathbb{R}_{++}^{N}$ are appropriately scaled so that $\xi_{\mathrm{L}}^{T} \xi_{\mathrm{R}}=1$ is satisfied.

(IV) The matrix $\mathcal{A}_{\mathrm{cl}}$ has eigenvalue 0 that is algebraically (and hence geometrically) simple. Moreover, we have $\operatorname{Re}(\lambda)<0\left(\forall \lambda \in \sigma\left(\mathcal{A}_{\mathrm{cl}}\right) \backslash\{0\}\right)$.

(V) We have

$$
\lim _{t \rightarrow \infty} \widehat{x}(t)=f(\widehat{x}(0)) \xi_{\mathrm{R}}, \quad f(\widehat{x}(0))=\xi_{\mathrm{L}}^{T} \widehat{x}(0)
$$

for any initial state $\widehat{x}(0) \in \mathbb{R}^{n_{\widehat{x}}}$.

The results (I), (III) and (V) of Theorem 5 clearly show that, under the conditions (i)-(iii), the interconnected system $\mathcal{G} \star \Omega$ has the property of persistence, and (20) in Definition 5 is satisfied with $\xi_{0}=\xi_{\mathrm{L}} \in \mathbb{R}_{++}^{n_{\widehat{x}}}$ and $\xi_{\infty}=\xi_{\mathrm{R}} \in \mathbb{R}_{++}^{n_{\widehat{x}}}$.

We need the following lemma for the proof of Theorem 5 . The proof of this lemma is given in the appendix section, Subsection IX-D.

Lemma 4: For given $A \in\left\{\mathbb{M}^{n \times n} \cap \mathbb{H}^{n \times n}\right\}, B \in \mathbb{R}_{+}^{n \times 1}$, and $C \in \mathbb{R}_{+}^{1 \times n}$, we have $A^{-1} B<0$ if $(A, B)$ is controllable. Similarly, we have $C A^{-1}<0$ if $(A, C)$ is observable.

\section{Proof of Theorem 5:}

Proof of (I): From (i) and Lemma 4, it is clear that $\overline{-C_{i} A_{i}^{-1} B_{i}}>0(i=1, \cdots, N)$. If we define $\mathcal{S}:=$ $\operatorname{diag}\left(-C_{1} A_{1}^{-1} B_{1}, \cdots,-C_{N} A_{N}^{-1} B_{N}\right) \in \mathbb{D}_{++}^{N \times N}$, we have $\mathcal{D}=\Psi-\mathcal{S}$. On the other hand, from (ii), (iii), and Proposition 3, we see that there exists $v_{\mathrm{R}} \in \mathbb{R}_{++}^{N}$ such that $\Psi \Omega v_{\mathrm{R}}=v_{\mathrm{R}}$. Note that $\Psi \Omega$ is irreducible if and only if $\Omega$ is since $\Psi \in \mathbb{D}_{++}^{N \times N}$. Therefore we have $(\mathcal{D} \Omega-I) v_{\mathrm{R}}=$ $((\Psi-\mathcal{S}) \Omega-I) v_{\mathrm{R}}=-\mathcal{S} \Omega v_{\mathrm{R}}=-\mathcal{S} \Psi^{-1} v_{\mathrm{R}}<0$. It follows from (the dual version of) (iii) of Lemma 1 that $\mathcal{D} \Omega-I$ is Hurwitz stable.

Proof of (II): From Theorem 3, we see that $\sigma\left(\mathcal{A}_{\mathrm{cl}}\right) \subset \mathbb{C}_{-}$if and only if $\lambda_{\mathrm{F}}(\Psi \Omega)<1$. Since at present $\lambda_{\mathrm{F}}(\Psi \Omega)=1$ holds from (iii), we see that $\sigma\left(\mathcal{A}_{\mathrm{cl}}\right) \subset \overline{\mathbb{C}_{-}}$holds from the continuity of the eigenvalue of $\mathcal{A}_{\mathrm{cl}}$ with respect to perturbations on it. Proof of (III): By defining $\Omega_{\mathcal{D}}:=\Omega(I-\mathcal{D} \Omega)^{-1}$, we readily see 


$$
\begin{aligned}
\mathcal{A}_{\mathrm{cl}} \xi_{\mathrm{R}} & =-\left(\mathcal{A}+\mathcal{B} \Omega_{\mathcal{D}} \mathcal{C}\right) \mathcal{A}^{-1} \mathcal{B} \Psi^{-1} v_{\mathrm{R}} \\
& =-\mathcal{B} \Psi^{-1} v_{\mathrm{R}}-\mathcal{B} \Omega_{\mathcal{D}} \mathcal{C} \mathcal{A}^{-1} \mathcal{B} \Psi^{-1} v_{\mathrm{R}} \\
& =-\mathcal{B} \Psi^{-1} v_{\mathrm{R}}-\mathcal{B} \Omega_{\mathcal{D}}(\mathcal{D}-\Psi) \Psi^{-1} v_{\mathrm{R}} \\
& =-\mathcal{B}\left(I+\Omega(I-\mathcal{D} \Omega)^{-1}(\mathcal{D}-\Psi)\right) \Psi^{-1} v_{\mathrm{R}} \\
& =-\mathcal{B}\left(I+(I-\Omega \mathcal{D})^{-1} \Omega(\mathcal{D}-\Psi)\right) \Psi^{-1} v_{\mathrm{R}} \\
& =-\mathcal{B}(I-\Omega \mathcal{D})^{-1}(I-\Omega \Psi) \Psi^{-1} v_{\mathrm{R}} \\
& =-\mathcal{B}(I-\Omega \mathcal{D})^{-1} \Psi^{-1}(I-\Psi \Omega) v_{\mathrm{R}} \\
& =0 .
\end{aligned}
$$

The equality $\xi_{\mathrm{L}}^{T} \mathcal{A}_{c l}=0$ follows similarly. On the other hand, since $\left(A_{i}, B_{i}\right)$ is controllable and $\left(A_{i}, C_{i}\right)$ is observable, we see $-A_{i}^{-1} B_{i}>0$ and $-C_{i} A_{i}^{-1}>0(i=1, \cdots, N)$ from Lemma 4. Moreover, since $\Psi \Omega$ is irreducible, we have $v_{\mathrm{R}}>0$ and $v_{\mathrm{L}}>0$ from Proposition 3. Therefore we have $\xi_{\mathrm{R}}=-\mathcal{A}^{-1} \mathcal{B} \Psi^{-1} v_{\mathrm{R}} \in \mathbb{R}_{++}^{n_{\widehat{x}}}$ and $\xi_{\mathrm{L}}=-\mathcal{A}^{-T} \mathcal{C}^{T} v_{\mathrm{L}} \in \mathbb{R}_{+{ }_{+}}^{n_{\widehat{x}}}$. Proof of (IV): We can prove that $\mathcal{A}_{\mathrm{cl}}$ is irreducible and hence the assertion readily follows from (II), (III) and Corollary 2. The proof for the irreducibility of $\mathcal{A}_{\mathrm{cl}}$, which is indeed the core of the proof of Theorem 5, is given in the appendix section, Subsection IX-E.

Proof of (V): Since (IV) holds and since $\xi_{\mathrm{R}} \in \mathbb{R}_{++}^{n_{\widehat{x}}}$ is the right eigenvector of $\mathcal{A}_{\mathrm{cl}}$ corresponding to the eigenvalue 0 , it is an elementary fact that the state $\widehat{x}$ of the interconnected system $\mathcal{G} \star \Omega$ converges to $f(\widehat{x}(0)) \xi_{\mathrm{R}}$ for some linear function $f: \mathbb{R}^{n_{\widehat{x}}} \rightarrow \mathbb{R}$. Furthermore, for the dynamics of the interconnected system represented by $\dot{\widehat{x}}=\mathcal{A}_{\mathrm{cl}} \widehat{x}$, we can readily see that $\xi_{\mathrm{L}}^{T} \dot{\hat{x}}=0$. Therefore we have $\xi_{\mathrm{L}}^{T} \widehat{x}(0)=f(\widehat{x}(0)) \xi_{\mathrm{L}}^{T} \xi_{\mathrm{R}}$. Since $\xi_{\mathrm{L}}^{T} \xi_{\mathrm{R}}=1$ from (III), we have $f(\widehat{x}(0))=\xi_{\mathrm{L}}^{T} \widehat{x}(0)$. This completes the proof.

In Theorem 5, the controllability and observability condition (i) is a natural requirement in system realization, and the irreducibility (i.e., the strong connectivity) of the interconnection matrix in condition (ii) is frequently assumed in the study of dynamical systems connected by network. Theorem 5 shows that, under these natural conditions as well as the stability boundary condition (iii), the interconnected positive system $\mathcal{G} \star \Omega$ naturally has the property of persistence.

\section{B. Analysis of Steady-State Output}

The next result concerns the steady state output of $\mathcal{G} \star \Omega$. This is a direct consequence of Theorem 5 and illustrates its usefulness in the application to formation control of multiagent systems with positive dynamics.

Theorem 6: Consider the case where every stable subsystem $G_{i}$ represented by (9) is SISO and satisfies the condition (i) in Theorem 5. Moreover, for given $v_{\text {obj }} \in \mathbb{R}_{++}^{N}$, assume that the interconnection matrix $\Omega \in \mathbb{R}_{+}^{N \times N}$ has the following property in addition to (ii) of Theorem 5:

(iii') $\Psi \Omega v_{\text {obj }}=v_{\text {obj }}$ holds.

Then, the output of the interconnected system $\mathcal{G} \star \Omega$ satisfies

$$
\lim _{t \rightarrow \infty} \widehat{z}(t)=f(\widehat{x}(0)) v_{\mathrm{obj}}\left(=\left(\xi_{\mathrm{L}}^{T} \widehat{x}(0)\right) v_{\mathrm{obj}}\right) \text {. }
$$

Here, $\xi_{\mathrm{L}} \in \mathbb{R}_{++}^{n_{\widehat{x}}}$ is given by (21) with $v_{\mathrm{L}} \in \mathbb{R}_{++}^{N}$ that satisfies $v_{\mathrm{L}} \Psi \Omega=v_{\mathrm{L}}$ and $\xi_{\mathrm{L}}^{T} \xi_{\mathrm{R}}=1$ for $\xi_{\mathrm{R}}=-\mathcal{A}^{-1} \mathcal{B} \Psi^{-1} v_{\text {obj }} \in \mathbb{R}_{++}^{n_{\widehat{x}}}$.

This theorem implies that, for a given $v_{\text {obj }} \in \mathbb{R}_{++}^{N}$ that represents the output position of each agent in a "desired formation," we can achieve the convergence (23) as long as we design the interconnection matrix $\Omega$ satisfying (ii) and (iii'). We emphasize that such synthesis of $\Omega$ can be done by solving linear equations (see Subsection IV-E for details). This is the basic idea to use the results in Theorems 5 and 6 for the formation control of multi-agent systems.

A brief sketch of the proof of Theorem 6 is as follows. Since $\Psi \Omega \in \mathbb{R}_{+}^{N \times N}$ satisfies $\Psi \Omega v_{\text {obj }}=v_{\text {obj }}$ for $v_{\text {obj }} \in \mathbb{R}_{++}^{N}$, we can see from Corollary 8.1.30 of [19] that $\lambda_{\mathrm{F}}(\Psi \Omega)=1$. Namely, a matrix $\Omega$ satisfying the condition (iii') satisfies the condition (iii) in Theorem 5 as well. It follows from Theorem 5 that $\widehat{x}_{\infty}=-f(\widehat{x}(0)) \mathcal{A}^{-1} \mathcal{B} \Psi^{-1} v_{\text {obj }}$ where $\widehat{x}_{\infty}:=\lim _{t \rightarrow \infty} \widehat{x}(t)$. Therefore, for $\widehat{z}_{\infty}:=\lim _{t \rightarrow \infty} \widehat{z}(t)$, we obtain

$$
\begin{aligned}
\widehat{z}_{\infty} & =(I-\mathcal{D} \Omega)^{-1} \mathcal{C} \widehat{x}_{\infty} \\
& =-f(\widehat{x}(0))(I-\mathcal{D} \Omega)^{-1} \mathcal{C} \mathcal{A}^{-1} \mathcal{B} \Psi^{-1} v_{\mathrm{obj}} \\
& =-f(\widehat{x}(0))(I-\mathcal{D} \Omega)^{-1}(\mathcal{D}-\Psi) \Psi^{-1} v_{\mathrm{obj}} \\
& =-f(\widehat{x}(0))(I-\mathcal{D} \Omega)^{-1}(\mathcal{D} \Omega-I) v_{\mathrm{obj}} \\
& =f(\widehat{x}(0)) v_{\mathrm{obj}}
\end{aligned}
$$

This validates the assertion in Theorem 6 .

\section{Extensions to Reducible Interconnection Matrices}

In this subsection, we extend the results in Theorems 5 and 6 to the case where the interconnection matrix $\Omega$ is reducible. To this end, suppose $\Omega \in \mathbb{R}_{+}^{N \times N}$ is of the form

$$
\Omega=\left[\begin{array}{cc}
\Omega_{\mathrm{u}} & \Omega_{\mathrm{ul}} \\
0 & \Omega_{\mathrm{l}}
\end{array}\right], \Omega_{\mathrm{u}} \in \mathbb{R}_{+}^{N_{\mathrm{u}} \times N_{\mathrm{u}}}, \Omega_{\mathrm{l}} \in \mathbb{R}_{+}^{N_{\mathrm{l}} \times N_{\mathrm{l}}}
$$

where $N_{\mathrm{u}}+N_{\mathrm{l}}=N$ and $\Omega_{\mathrm{l}}$ is scalar 0 or irreducible. Note that, from Definition 6 , any reducible matrix $\Omega \in \mathbb{R}_{+}^{N \times N}$ can be reduced into this form by a transformation with a permutation matrix. According to the partition (24), we let

$$
\begin{aligned}
& \mathcal{G}=\operatorname{diag}\left(\mathcal{G}_{\mathrm{u}}, \mathcal{G}_{\mathrm{l}}\right), \quad \Psi=\operatorname{diag}\left(\Psi_{\mathrm{u}}, \Psi_{\mathrm{l}}\right), \\
& \mathcal{A}=\operatorname{diag}\left(\mathcal{A}_{\mathrm{u}}, \mathcal{A}_{\mathrm{l}}\right), \quad \mathcal{B}=\operatorname{diag}\left(\mathcal{B}_{\mathrm{u}}, \mathcal{B}_{\mathrm{l}}\right), \\
& \mathcal{C}=\operatorname{diag}\left(\mathcal{C}_{\mathrm{u}}, \mathcal{C}_{\mathrm{l}}\right), \quad \mathcal{D}=\operatorname{diag}\left(\mathcal{D}_{\mathrm{u}}, \mathcal{D}_{\mathrm{l}}\right), \\
& \widehat{x}=\left[\begin{array}{ll}
\widehat{x}_{\mathrm{u}}^{T} & \widehat{x}_{\mathrm{l}}^{T}
\end{array}\right]^{T} \in \mathbb{R}^{n_{\widehat{x}}}, \\
& \widehat{x}_{\mathrm{u}} \in \mathbb{R}^{n_{\widehat{x}_{\mathrm{u}}}}, \widehat{x}_{1} \in \mathbb{R}^{n_{\widehat{x}_{1}}}, n_{\widehat{x}_{\mathrm{u}}}:=\sum_{i=1}^{N_{\mathrm{u}}} n_{i}, n_{\widehat{x}_{1}}:=\sum_{i=N_{\mathrm{u}}+1}^{N} n_{i}, \\
& \widehat{z}=\left[\begin{array}{cc}
\widehat{z}_{\mathrm{u}}^{T} & \widehat{z}_{\mathrm{l}}^{T}
\end{array}\right]^{T} \in \mathbb{R}^{N}, \widehat{z}_{\mathrm{u}} \in \mathbb{R}^{N_{\mathrm{u}}}, \widehat{z}_{\mathrm{l}} \in \mathbb{R}^{N_{\mathrm{l}}} .
\end{aligned}
$$

Then, we can readily obtain the following corollary from Theorems 5 and 6.

Corollary 3: Consider the case where every stable subsystem $G_{i}$ represented by (9) is SISO and satisfies the condition (i) in Theorem 5. Moreover, assume that the interconnection matrix $\Omega \in \mathbb{R}_{+}^{N \times N}$ is given of the form (24) and

(ii-a) $\Omega_{1} \in \mathbb{R}_{+}^{N_{1} \times N_{1}}$ is irreducible,

(ii-b) $\left(\Psi_{\mathrm{u}} \Omega_{\mathrm{u}}+I\right)^{N_{\mathrm{u}}-1} \Omega_{\mathrm{ul}} \in \mathbb{R}_{++}^{N_{\mathrm{u}} \times N_{\mathrm{l}}}$ holds, and

(iii') $\Psi \Omega v_{\text {obj }}=v_{\text {obj }}$ holds for given $v_{\text {obj }} \in \mathbb{R}_{++}^{N}$ with $v_{\text {obj }}=$ $\left[v_{\text {obj }, \mathrm{u}}^{T} v_{\text {obj }, 1}^{T}\right]^{T}\left(v_{\text {obj }, \mathrm{u}} \in \mathbb{R}_{++}^{N_{\mathrm{u}}}, v_{\mathrm{obj}, \mathrm{l}} \in \mathbb{R}_{++}^{N_{1}}\right)$.

Then, the output of the interconnected system $\mathcal{G} \star \Omega$ satisfies $\lim _{t \rightarrow \infty} \widehat{z}(t)=f_{\mathrm{l}}\left(\widehat{x}_{1}(0)\right) v_{\mathrm{obj}}\left(=\left(\xi_{\mathrm{L}, \mathrm{l}}^{T} \widehat{x}_{1}(0)\right) v_{\mathrm{obj}}\right)$.

Here, $\xi_{\mathrm{L}, 1} \in \mathbb{R}_{++}^{n_{\widehat{x}_{1}}}$ is given by $\xi_{\mathrm{L}, \mathrm{l}}=-\mathcal{A}_{\mathrm{l}}^{-T} \mathcal{C}_{\mathrm{l}}^{T} v_{\mathrm{L}, \mathrm{l}}$ with $v_{\mathrm{L}, 1} \in$ $\mathbb{R}_{++}^{N_{1}}$ that satisfies $v_{\mathrm{L}, 1} \Psi_{1} \Omega_{1}=v_{\mathrm{L}, 1}$ and $\xi_{\mathrm{L}, 1}^{T} \xi_{\mathrm{R}, 1}=1$ for $\xi_{\mathrm{R}, \mathrm{l}}=$ $-\mathcal{A}_{1}^{-1} \mathcal{B}_{\mathrm{l}} \Psi_{1}^{-1} v_{\mathrm{obj}, \mathrm{l}} \in \mathbb{R}_{++}^{n_{\widehat{x}_{1}}}$. 
Proof of Corollary 3: Note that, under the partition (24) and (25), the interconnected system $G \star \Omega$ can be represented by Fig. 3. With this in mind, let us rewrite the condition (iii') as

$$
\left[\begin{array}{cc}
\Psi_{\mathrm{u}} & 0 \\
0 & \Psi_{1}
\end{array}\right]\left[\begin{array}{cc}
\Omega_{\mathrm{u}} & \Omega_{\mathrm{ul}} \\
0 & \Omega_{\mathrm{l}}
\end{array}\right]\left[\begin{array}{c}
v_{\mathrm{obj}, \mathrm{u}} \\
v_{\mathrm{obj}, 1}
\end{array}\right]=\left[\begin{array}{c}
v_{\mathrm{obj}, \mathrm{u}} \\
v_{\mathrm{obj}, 1}
\end{array}\right] .
$$

This specifically shows that $\Psi_{1} \Omega_{1} v_{\mathrm{obj}, 1}=v_{\mathrm{obj}, 1}$ holds. Since $\Omega_{1}$ is assumed to be irreducible as in (ii-a), we see from Theorem 6 that $\lim _{t \rightarrow \infty} \widehat{z}_{1}(t)=f_{1}\left(\widehat{x}_{1}(0)\right) v_{\mathrm{obj}, 1}\left(=: \widehat{z}_{1, \infty}\right)$. On the other hand, (27) shows $\left(\Psi_{\mathrm{u}} \Omega_{\mathrm{u}}-I\right) v_{\mathrm{obj}, \mathrm{u}}=-\Omega_{\mathrm{ul}} v_{\mathrm{obj}, \mathrm{l}}$. Here, if we define $g:=\left(\Psi_{\mathrm{u}} \Omega_{\mathrm{u}}+I\right)^{N_{\mathrm{u}}-1} v_{\mathrm{obj}, \mathrm{u}}$, we see from (ii-b) and $v_{\mathrm{obj}, \mathrm{u}} \in \mathbb{R}_{++}^{N_{\mathrm{u}}}$ that $g \in \mathbb{R}_{++}^{N_{\mathrm{u}}}$. Moreover,

$$
\begin{aligned}
\left(\Psi_{\mathrm{u}} \Omega_{\mathrm{u}}-I\right) g & =\left(\Psi_{\mathrm{u}} \Omega_{\mathrm{u}}-I\right)\left(\Psi_{\mathrm{u}} \Omega_{\mathrm{u}}+I\right)^{N_{\mathrm{u}}-1} v_{\mathrm{obj}, \mathrm{u}} \\
& =\left(\Psi_{\mathrm{u}} \Omega_{\mathrm{u}}+I\right)^{N_{\mathrm{u}}-1}\left(\Psi_{\mathrm{u}} \Omega_{\mathrm{u}}-I\right) v_{\mathrm{obj}, \mathrm{u}} \\
& =-\left(\Psi_{\mathrm{u}} \Omega_{\mathrm{u}}+I\right)^{N_{\mathrm{u}}-1} \Omega_{\mathrm{ul}} v_{\mathrm{obj}, \mathrm{l}} \\
& <0
\end{aligned}
$$

where we again use (ii-b) to ensure the last strict inequality. It follows from (the dual version of) (iii) of Lemma 1 that $\Psi_{\mathrm{u}} \Omega_{\mathrm{u}}-I$ is Hurwitz stable. Therefore, we see from Theorem 3 that the interconnected system $\mathcal{G}_{\mathrm{u}} \star \Omega_{\mathrm{u}}$ is admissible and stable. To summarize, in Fig. 3, the stable interconnected system $\left(\mathcal{G}_{\mathrm{u}} \star \Omega_{\mathrm{u}}\right) \Omega_{\mathrm{ul}}$ receives the input signal $\widehat{z}_{1, \infty}$ at the steady state. Thus the proof is completed if we show that, under (27), the output of $\left(\mathcal{G}_{\mathrm{u}} \star \Omega_{\mathrm{u}}\right) \Omega_{\mathrm{ul}}$ with respect to the step input $\widehat{z}_{1, \infty}=f_{1}\left(\widehat{x}_{1}(0)\right) v_{\mathrm{obj}, 1}$ satisfies

$$
\lim _{t \rightarrow \infty} \widehat{z}_{\mathrm{u}}(t)=f_{\mathrm{l}}\left(\widehat{x}_{\mathrm{l}}(0)\right) v_{\mathrm{obj}, \mathrm{u}} .
$$

The proof of this part is elementary and given in the appendix section, Subsection IX-F.

Remark 5: The condition (ii-b) in Corollary 3 is not stringent. From Proposition 2, we see that this condition holds at least if $\Omega_{\mathrm{u}} \in \mathbb{R}_{+}^{N_{\mathrm{u}} \times N_{\mathrm{u}}}$ is irreducible and each column of $\Omega_{\mathrm{ul}} \in \mathbb{R}_{+}^{N_{\mathrm{u}} \times N_{1}}$ is non-zero.

Remark 6: Let us consider the case where $N_{\mathrm{l}}=1$, $\left(\mathcal{A}_{1}, \mathcal{B}_{1}, \mathcal{C}_{1}, \mathcal{D}_{1}\right)=(-1,1,1,0), \Omega_{1}=1$ and $\widehat{x}_{1}(0)=v_{\text {obj, }} \in$ $\mathbb{R}_{++}$(this essentially coincides with the case where $\mathcal{G}_{1} \star \Omega_{1}$ is an integrator $\dot{\widehat{x}}_{1}=0$ with the initial condition $\left.\widehat{x}_{1}(0)=v_{\mathrm{obj}, 1}\right)$. Then, since $\Psi_{1}=1$, it is clear that the second row of (27) is satisfied, and furthermore, we see $\widehat{z}_{1}(t) \equiv v_{\mathrm{obj}, 1}$ and $f_{1}\left(\widehat{x}_{1}(0)\right)=1$. In this case, Corollary 3 implies that, if a step input $v_{\mathrm{obj}, 1} \in \mathbb{R}_{++}$is applied to $\left(\mathcal{G}_{\mathrm{u}} \star \Omega_{\mathrm{u}}\right) \Omega_{\mathrm{ul}}$, we can let $\widehat{z}_{\mathrm{u}}$ track a given $v_{\mathrm{obj}, \mathrm{u}} \in \mathbb{R}_{++}^{N_{\mathrm{u}}}$ precisely if (ii-b) holds and

$$
\Psi_{\mathrm{u}}\left[\begin{array}{ll}
\Omega_{\mathrm{u}} & \Omega_{\mathrm{ul}}
\end{array}\right]\left[\begin{array}{c}
v_{\mathrm{obj}, \mathrm{u}} \\
v_{\mathrm{obj}, \mathrm{l}}
\end{array}\right]=v_{\mathrm{obj}, \mathrm{u}} .
$$

Note that this simple equality condition and the condition (ii-b) ensure the admissibility and stability of $\mathcal{G}_{\mathrm{u}} \star \Omega_{\mathrm{u}}$ as well as the satisfaction of the tracking requirement. We apply this result to an energy management problem in DC-grids in Section VI.

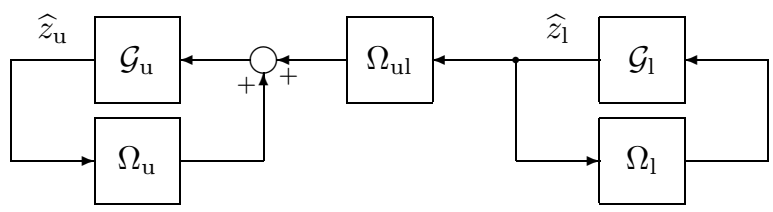

Fig. 3. Interconnected System $\mathcal{G} \star \Omega$ with partition (24) and (25).

\section{Relationship with the f-Consensus Protocol [27], [14]}

Theorems 5 and 6 are closely related to (and meaningful extensions of the results already obtained in the study area of multi-agent systems [14], [27], [38], [39]. In this section, we show that the $f$-consensus protocol shown in [27], [14] can readily be obtained along with Theorem 5 .

The communication over multi-agents in [27], [14] is determined by the directed graph $G(\mathcal{I}, \mathcal{E})$ with the set of nodes $\mathcal{I}:=\{1, \cdots, N\}$ and edges $\mathcal{E} \subseteq \mathcal{I} \times \mathcal{I}$. The dynamics of the agents are assumed to be identical integrators as in

$$
P_{i}: \dot{x}_{i}(t)=w_{i}(t), \quad x_{i}(t) \in \mathbb{R} .
$$

The goal is to determine the input $w_{i}(i=1, \cdots, N)$ by the communication with other agents over network so that we can achieve

$$
\lim _{t \rightarrow \infty} \widehat{x}(t)=f(\widehat{x}(0)) \mathbf{1}^{N}, \widehat{x}:=\left[x_{1}, \cdots, x_{N}\right]^{T} \in \mathbb{R}^{N} .
$$

If (31) is achieved for some $f: \mathbb{R}^{N} \rightarrow \mathbb{R}$, we say that $f$ consensus is achieved. In order to achieve an $f$-consensus, the following protocol is presented in [27], [14]:

$$
w_{i}(t)=\sum_{j \in \mathcal{N}_{i}}\left(x_{j}(t)-x_{i}(t)\right) .
$$

Here, $\mathcal{N}_{i}$ is the set of neighbors of the node $i$ defined by $\mathcal{N}_{i}:=$ $\{j \in \mathcal{I}:(j, i) \in \mathcal{E}\}$. The interconnected system constructed from (30) and (32) can be represented by

$$
\dot{\hat{x}}(t)=-L \widehat{x}(t),
$$

where $L \in \mathbb{R}^{N \times N}$ is the graph Laplacian of $G$ defined by

$$
\begin{aligned}
& L:=D-A, D:=\operatorname{diag}\left(d_{1}, \cdots, d_{N}\right), d_{i}=\left|\mathcal{N}_{i}\right|, \\
& A:=\left[A_{i, j}\right], A_{i, j}=1\left(j \in \mathcal{N}_{i}\right), A_{i, j}=0\left(j \notin \mathcal{N}_{i}\right) .
\end{aligned}
$$

It is easy to see that $L \mathbf{1}^{N}=0$ holds (i.e., $\mathbf{1}^{N} \in \mathbb{R}_{++}^{N}$ is the right-eigenvector of $L$ with respect to the eigenvalue 0 ). It is shown in [27], [14] that, if the graph $G(\mathcal{I}, \mathcal{E})$ is strongly connected, an $f$-consensus is achieved by (32) as in

$$
\lim _{t \rightarrow \infty} \widehat{x}(t)=f(\widehat{x}(0)) \mathbf{1}^{N}, \quad f(\widehat{x}(0))=\xi_{0}^{T} \widehat{x}(0) .
$$

Here, $\xi_{0} \in \mathbb{R}^{N}$ is the left-eigenvector of $L$ with respect to the eigenvalue 0 satisfying $\xi_{0}^{T} \mathbf{1}^{N}=1$.

In the following, we will show that (35) follows directly from Theorem 5. To this end, we first note that (33) is a positive system since $-L \in \mathbb{M}^{N \times N}$. Moreover, (33) can be rewritten as

$$
\dot{\hat{x}}(t)=-D \widehat{x}(t)+\widehat{w}(t), \widehat{z}(t)=\widehat{x}(t), \widehat{w}(t)=A \widehat{z}(t) .
$$

From this expression, we can regard (33) as an interconnected system constructed from $N$ positive, SISO and stable subsystems $G_{i}(i=1, \cdots, N)$ given by

$$
G_{i}:\left\{\begin{aligned}
\dot{x}_{i}(t) & =-d_{i} x_{i}(t)+w_{i}(t) \\
z_{i}(t) & =x_{i}(t)
\end{aligned}\right.
$$

and the interconnection matrix

$$
\Omega=A \in \mathbb{R}_{+}^{N \times N} .
$$

It is clear that $G_{i}(i=1, \cdots, N)$ in the form of (37) satisfies the condition (i) of Theorem 5. On the other hand, the interconnection matrix $\Omega \in \mathbb{R}_{+}^{N \times N}$ given in (38) is irreducible 
if and only if the graph $G(\mathcal{I}, \mathcal{E})$ is strongly connected. The Frobenius eigenvalue of $\Psi \Omega=D^{-1} A$ is 1 with the righteigenvector $v_{\mathrm{R}}=\mathbf{1}^{N} \in \mathbb{R}_{++}^{N}$ and the left-eigenvector $v_{\mathrm{L}}=D \xi_{0}$. Therefore $\Omega \in \mathbb{R}_{+}^{N \times N}$ satisfies the condition (ii) and (iii) of Theorem 5. Moreover, it is easy to see from (21) that $\xi_{\mathrm{R}}=\mathbf{1}^{N}$ and $\xi_{\mathrm{L}}=\xi_{0}$ in this case. It follows that (22) in Theorem 5 coincides with (35).

To summarize, Theorem 5 turns out to be an intriguing extension of $f$-consensus protocols shown in [27], [14]. Theorems 5 and 6 show that, under certain conditions, we can achieve $f$-consensus (with respect to the output of each subsystem) even if we generalize the dynamics of each agent from integrators to positive systems, and interconnection matrix from graph-Laplacian matrices to nonnegative matrices.

\section{E. Parametrization of Interconnection Matrices}

For the preparation of formation control of multi-agent systems based on Theorems 5 and 6 , it is meaningful to show a concrete way to construct a desired $\Omega \in \mathbb{R}_{+}^{N \times N}$ that satisfies $\Psi \Omega v_{\text {obj }}=v_{\text {obj }}$ and $\Gamma(\Omega)=\Gamma$ for prescribed $v_{\text {obj }} \in \mathbb{R}_{++}^{N}$ and graph structure $\Gamma$. For illustration, consider the cases where $\Gamma$ is shown in Figs. 4 and 5 for $N=3$.

For graph structure $\Gamma_{\mathrm{A}}$, any interconnection matrix $\Omega \in$ $\mathbb{R}_{+}^{N \times N}$ satisfying $\Psi \Omega v_{\text {obj }}=v_{\text {obj }}$ and $\Gamma(\Omega)=\Gamma_{\mathrm{A}}$ can be parametrized by

$$
\Omega=\Psi^{-1} \Omega\left(v_{\mathrm{obj}}, p\right) \in \mathbb{R}_{++}^{N \times N}
$$

where

$$
\begin{aligned}
& \Omega\left(v_{\mathrm{obj}}, p\right)_{i, j}= \\
& \begin{cases}\left(1-p_{1}\right) \frac{v_{\mathrm{obj}, 1}}{v_{\mathrm{obj}, N}} & (i, j)=(1, N), \\
p_{i} \frac{v_{\mathrm{obj}, i}}{v_{\mathrm{obj}, j}} & (1 \leq i \leq N, j=i+1), \\
\left(1-p_{i}\right) \frac{v_{\mathrm{obj}, i}}{v_{\mathrm{obj}, j}} & (1 \leq i \leq N, j=i-1), \\
p_{N} \frac{v_{\mathrm{obj}, N}}{v_{\mathrm{obj}, 1}} & (i, j)=(N, 1), \\
0 & \text { otherwise. }\end{cases}
\end{aligned}
$$

Here, parameter $p \in \mathbb{R}_{++}^{N}$ can be chosen arbitrarily among $0<p<\mathbf{1}^{N}$. On the other hand, for graph structure $\Gamma_{\mathrm{B}}$, any interconnection matrix $\Omega \in \mathbb{R}_{+}^{N \times N}$ satisfying $\Psi \Omega v_{\text {obj }}=v_{\text {obj }}$ and $\Gamma(\Omega)=\Gamma_{\mathrm{B}}$ can be parametrized again by (39) and (40) where parameter $p \in \mathbb{R}_{++}^{N}$ can be chosen such that $p_{1}=$ $1, p_{N}=0$, and $0<p_{i}<1(i=2, \cdots, N-1)$. In both cases, we can confirm that resulting interconnection matrix $\Omega$ is irreducible (since $\Gamma_{\mathrm{A}}$ and $\Gamma_{\mathrm{B}}$ are both strongly connected).

Remark 7: The parametrization (39) and (40) have been derived based on the fact that the linear equation $\Psi \Omega v_{\mathrm{obj}}=v_{\mathrm{obj}}$ can be solved row by row as in $G_{i}(0) \sum_{j \in \mathcal{N}_{i}} \Omega_{i, j} v_{\mathrm{obj}, j}=$ $v_{\mathrm{obj}, i}(i=1, \cdots, N)$, where $\mathcal{N}_{i}$ is the set of neighbors of

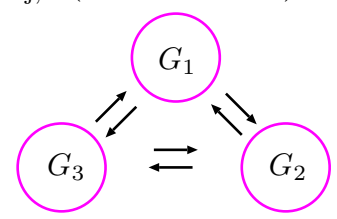

Fig. 4. Graph structure $\Gamma_{\mathrm{A}}$.

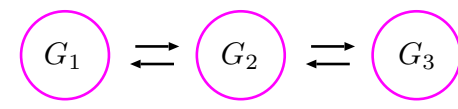

Fig. 5. Graph structure $\Gamma_{\mathrm{B}}$. subsystem $i$ defined by $\mathcal{N}_{i}:=\left\{j: \Omega_{i, j}>0\right.$ is allowed $\}$. This further implies that in general the calculation of $\Omega$ can be done in a distributed way in the sense that $\Omega_{i, j}\left(j \in \mathcal{N}_{i}\right)$ needed for the subsystem $i$ can be computed if $v_{\mathrm{obj}, j}\left(j \in \mathcal{N}_{i} \cup i\right)$ are available. Moreover, since the condition $\Psi \Omega v_{\mathrm{obj}}=v_{\mathrm{obj}}$ depends only on the steady-state gain $\Psi$, we can design $\Omega$ without precise information of the subsystems $G_{i}(i=1, \cdots, N)$. It is also true that the resulting $\Omega$ robustly achieves the desired formation as long as perturbations on each subsystem do not affect its steady-state gain.

\section{ApPlication to Formation CONTROL OF Multi-Agent Positive Systems}

In this section, we apply the results in Section IV to formation control of multi-agent systems.

\section{A. Problem Setting and Consensus-based Formation Control}

Let us consider a multi-agent system with $N$ agents, where the $i$-th agent $(i=1, \cdots, N)$ can move over the $(x, y)$ plane. We denote by $\left(z_{i, x}(t), z_{i, y}(t)\right)$ the position of agent $i$. Furthermore, we define $\widehat{z}_{j}:=\left[\begin{array}{lll}z_{1, j} & \cdots & z_{N, j}\end{array}\right]^{T}(j=x, y)$ by stacking the coordinates of the agents.

We assume that agent $i$ has independent dynamics along the $x$ - and $y$-axes, denoted by $P_{i, x}(s)$ and $P_{i, y}(s)$, respectively, and independent control inputs $u_{i, x}(t)$ and $u_{i, y}(t)$. We further assume that, as typical dynamics of moving agents, $P_{i, j}(s)$ are given by

$$
\begin{aligned}
& Z_{i, j}(s)=P_{i, j}(s) U_{i, j}(s), \quad P_{i, j}(s)=\frac{k_{i, j}}{s\left(s+a_{i, j}\right)}, \\
& k_{i, j}>0, a_{i, j}>0(i=1, \cdots, N, j=x, y) .
\end{aligned}
$$

Since $P_{i, j}(s)$ is not stable (or say, on the stability boundary), we cannot apply directly the results in Theorem 6. To get around this difficulty, we apply a minor feedback as in

$$
u_{i, j}(t)=-f_{i, j}\left(z_{i, j}(t)-w_{i, j}(t)\right)(i=1, \cdots, N, j=x, y)
$$

with $0<f_{i, j} \leq a_{i, j}^{2} / 4 k_{i, j}$, where $w_{i, j}(i=1, \cdots, N, j=$ $x, y)$ is the exogenous input kept for the interconnection. Then we have

$$
\begin{aligned}
& Z_{i, j}(s)=G_{i, j}(s) W_{i, j}(s), \\
& G_{i, j}(s)=\left[\begin{array}{cc|c}
-b_{i, j} & 1 & 0 \\
0 & -c_{i, j} & b_{i, j} c_{i, j} \\
\hline 1 & 0 & 0
\end{array}\right], \\
& b_{i, j}+c_{i, j}=a_{i, j}, \quad b_{i, j} c_{i, j}=f_{i, j} k_{i, j} .
\end{aligned}
$$

It follows from Proposition 1 that $G_{i, j}(i=1, \cdots, N, j=$ $x, y)$ are positive (with respect to the minimal realizations (41)), SISO, and stable systems with $G_{i, j}(0)=1(i=$ $1, \cdots, N, j=x, y)$. The last property is a natural consequence from the fact that each open-loop transfer function $P_{i, j}(s)(i=1, \cdots, N, j=x, y)$ includes an integrator. We emphasize that the properties of $G_{i, j}(s)$ mentioned above robustly hold against "small" perturbations on the plant parameters and the minor-feedback gains. For description simplicity, we define $\widehat{w}_{j}:=\left[\begin{array}{lll}w_{1, j} & \cdots & w_{N, j}\end{array}\right]^{T}(j=x, y)$.

We assume that $N$-agents independently communicate their $x$ and $y$ positions each other. Our goal here is to design interconnection matrices $\Omega_{x}$ and $\Omega_{y}$ such that, under the 
interconnection with $\Omega_{x}$ and $\Omega_{y}$ for $\left(\widehat{z}_{x}, \widehat{w}_{x}\right)$ and $\left(\widehat{z}_{y}, \widehat{w}_{y}\right)$, respectively, the following formation can be achieved:

$$
\left.\lim _{t \rightarrow \infty}\left[\widehat{z}_{x}(t) \widehat{z}_{y}(t)\right)\right]=\left[f_{x}\left(\widehat{x}_{x}(0)\right) v_{\mathrm{obj}, x} f_{y}\left(\widehat{x}_{y}(0)\right) v_{\mathrm{obj}, y}\right] .
$$

Here, $v_{\mathrm{obj}, j} \in \mathbb{R}_{++}^{N}(j=x, y)$ are given vectors that specify the desired formation, and $\widehat{x}_{j}(0)(j=x, y)$ stand for the initial states of the corresponding interconnected systems. On the other hand, $f_{j}: \mathbb{R}^{2 N} \rightarrow \mathbb{R}(j=x, y)$ stand for the scaling factors. It is obvious that we can readily solve this problem by following Theorem 6 .

Remark 8: Since the synthesis method of the interconnection matrices proposed in Theorem 6 is based on the idea of consensus, and since we do not allow to incorporate any external signals to the interconnected systems, we cannot exclude the effect of initial states at the limits of the outputs. The problem setting (42) has been defined keeping this fact in mind. Similar problem setting can be found in [40].

Remark 9: To illustrate our results in Theorem 6 in a realistic situation, we assumed typical second-order dynamics of moving agents (i.e., integrator plus first-order lag) and showed that we can make them positive and stable by applying minorfeedbacks. For those cases where the dynamics of moving agents are of higher-order, however, retrieving positivity and stability by minor-feedback becomes hard. This poses essential limitation on the application of Theorem 6 to such cases.

\section{B. Numerical Examples}

Along with the basic problem settings stated in Subsection V-A, we generated $a_{i, j}$ and $k_{i, j}$ randomly over the closed interval [ $\left[\begin{array}{ll}10 & 20\end{array}\right]$ and [ $\left[\begin{array}{ll}1 & 2\end{array}\right]$, respectively, and then let $f_{i, j}$ as $f_{i, j}=0.8 \times a_{i, j}^{2} / 4 k_{i, j}$. We thus constructed $G_{i, j}(i=1, \cdots, N, j=x, y)$. We let $\left[\begin{array}{ll}v_{\mathrm{obj}, x} & \left.v_{\mathrm{obj}, y}\right]_{i}=\end{array}\right.$ $[2+\cos (2 \pi i / N) 2+\sin (2 \pi i / N)]$ so that the agents can form a (scaled) circle. As for the graph structure of the interconnection matrices, we consider $\Gamma_{\mathrm{A}}$ (see Fig. 4). Namely, we designed $\left(\Omega_{x}^{[\mathrm{A}]}, \Omega_{y}^{[\mathrm{A}]}\right)$ with $\Gamma\left(\Omega_{j}^{[A]}\right)=\Gamma_{\mathrm{A}}(j=x, y)$ by the parametrization shown in (40) with $p_{i}=1 / 2(i=1, \cdots, N)$.

Figs 6-9 are the simulation results for the case $N=20$.

We see that the agents gradually form a (scaled) circle and converge to the position shown by blue dot which is computed in advance from (23).

Remark 10: In the case where subsystems are homogeneous and hence $G_{1}(0)=\cdots=G_{N}(0)=: \gamma$ holds, we see from Theorem 6 that the desired formation is achieved if $\Omega v_{\text {obj }}=(1 / \gamma) v_{\text {obj }}$. Namely, the achievement of the formation solely depends on the Frobenius eigenvalue $\lambda_{\mathrm{F}}(\Omega)=1 / \gamma$ and its associated right eigenvector and other eigenvalues of $\Omega$ are not relevant. However, the location of the eigenvalues of $\Omega$ strongly affects the speed of convergence. In particular, if the interconnection matrix $\Omega$ has real eigenvalues only, it has been shown that the second largest positive eigenvalue (next to $\lambda_{\mathrm{F}}(\Omega)$ ) is a key factor in determining the speed of convergence. See [11] for details. Note that this result conforms to the well-known fact that, in $f$-consensus protocol discussed in Subsection IV-D, the second smallest eigenvalue of the graph Laplacian determines the speed of convergence of consensus (this eigenvalue is often called algebraic connectivity [27]).

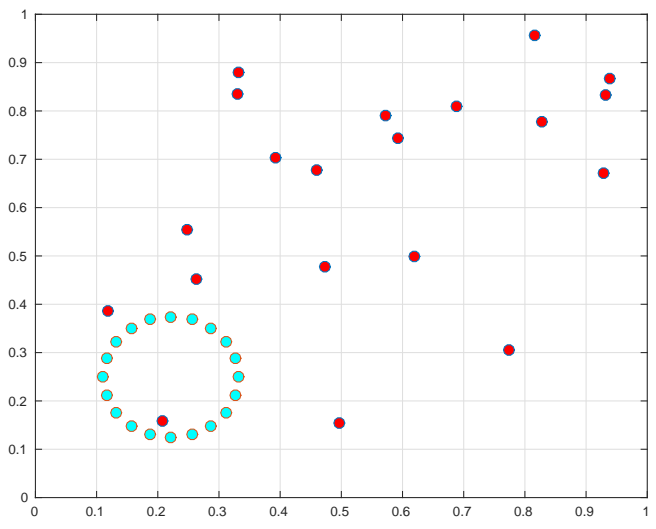

Fig. 6. Agent position under $\left(\Omega_{x}^{[\mathrm{A}]}, \Omega_{y}^{[\mathrm{A}]}\right)(t=0[\mathrm{sec}])$.

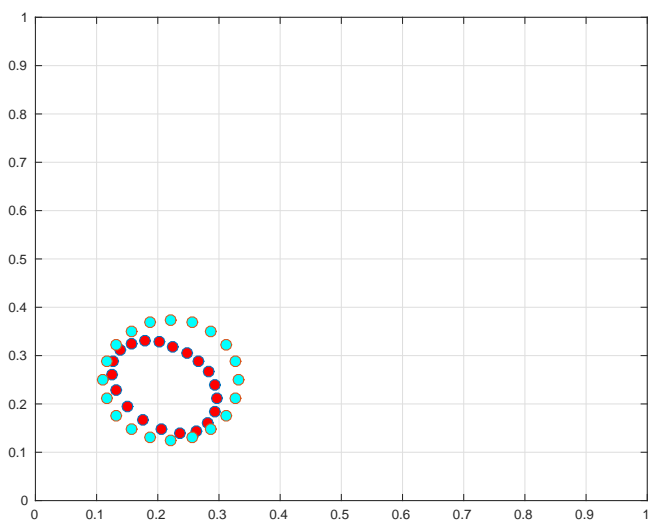

Fig. 7. Agent position under $\left(\Omega_{x}^{[\mathrm{A}]}, \Omega_{y}^{[\mathrm{A}]}\right)(t=10[\mathrm{sec}])$.

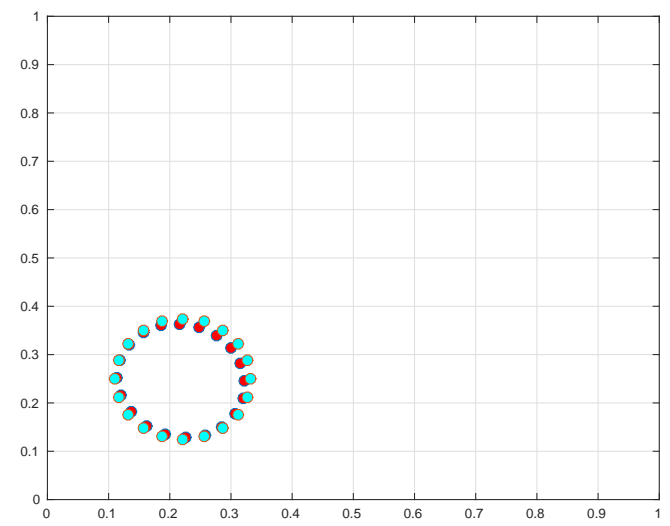

Fig. 8. Agent position under $\left(\Omega_{x}^{[\mathrm{A}]}, \Omega_{y}^{[\mathrm{A}]}\right)(t=20[\mathrm{sec}])$.

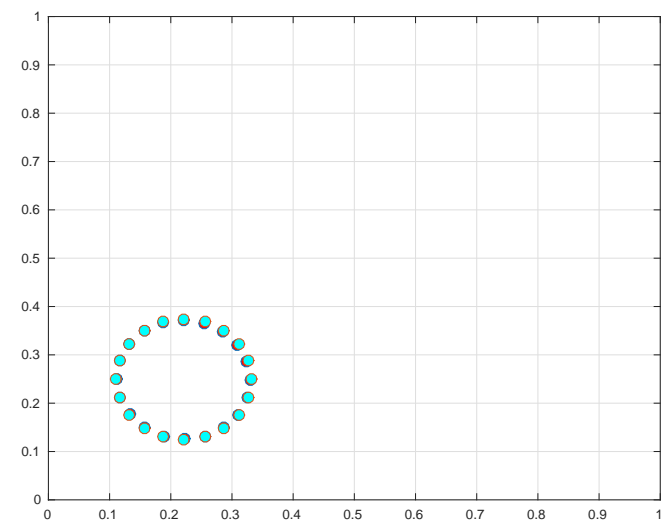

Fig. 9. Agent position under $\left(\Omega_{x}^{[\mathrm{A}]}, \Omega_{y}^{[\mathrm{A}]}\right)(t=30[\mathrm{sec}])$. 


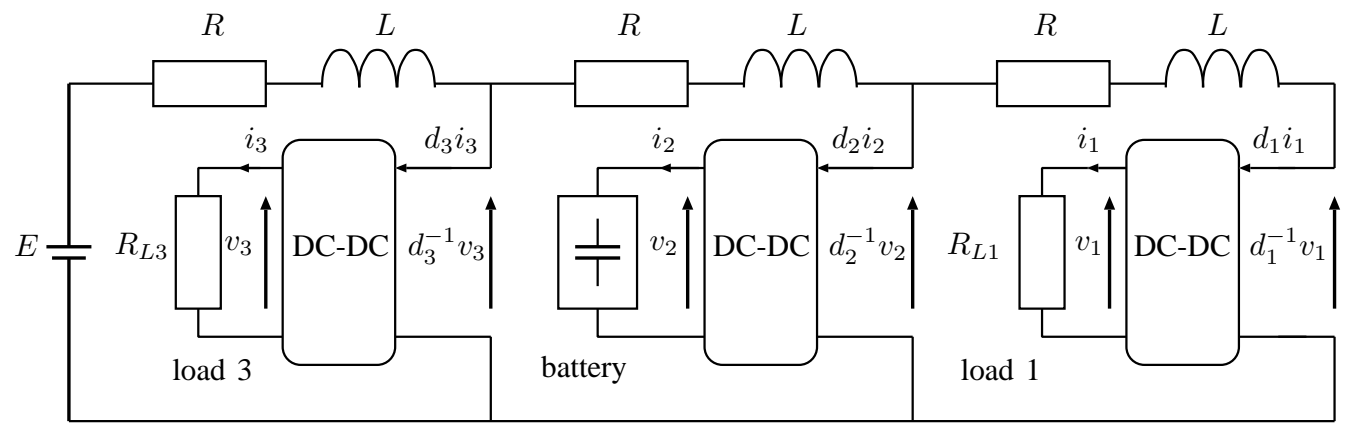

Fig. 10. A DC-grid composed of one DC voltage source, two loads, and one battery.

\section{Application to EnERgy ManAGEMENT IN DC-GRIDS}

Recently, intensive research effort has been made for analysis and synthesis of power-grid networks. In particular, DC (Direct Current)-grids are expected to play an important role in future power electronic systems, in view of recent drastic increases and technological developments in DC generation, DC storage tanks (such as lithium-ion rechargeable batteries and electric double-layer capacitors), and DC loads [2], [22], [24], [36]. In this section, we apply the theoretical result in Corollary 3 to an energy management problem in a DC-grid.

\section{A. DC-Grid and Problem Setting}

Let us consider the DC-grid shown in Fig. 10. This DCgrid is composed of DC voltage source with voltage $E$, transmission lines with resistance $R$ and inductance $L$, two DC loads with resistance $R_{L 1}$ and $R_{L 3}$, and a battery. The voltage and current of the load 1 , the battery, and the load 3 are denoted by $\left(v_{1}, i_{1}\right),\left(v_{2}, i_{2}\right)$, and $\left(v_{3}, i_{3}\right)$, respectively. We assume that the SOC (State of Charge) of the battery, denoted by $s$, is linear with respect to $v_{2}$ and given by $s=K v_{2}$ where $K$ is a given constant. As shown in Fig. 10, the load 1 , the battery, and the load 3 are equipped with ideal DC-DC converters with voltage gains $d_{1}, d_{2}$, and $d_{3}$, respectively.

The objective here is to supply desired voltages for the two loads and achieve a desired SOC of the battery at the steady state by appropriately determining the DC-DC converter gains $d_{1}, d_{2}$, and $d_{3}$. Namely, for given reference values $v_{1}^{\star}, s^{\star}$, and $v_{3}^{\star}$, the goal is to determine $d_{1}, d_{2}$, and $d_{3}$ such that

$$
\lim _{t \rightarrow \infty}\left[\begin{array}{lll}
v_{1}(t) & s(t) & v_{3}(t)
\end{array}\right]^{T}=\left[\begin{array}{lll}
v_{1}^{\star} & s^{\star} & v_{3}^{\star}
\end{array}\right]^{T} \text {. }
$$

Even though we might be able to approach this problem by standard electric circuit analysis, we demonstrate that the results in Corollary 3 and Remark 6 is effective for highly constructive treatment.

\section{B. Modeling as an Interconnected Positive System}

We assume that the dynamics of the battery can be modeled as $\dot{s}=i_{2}$. Then the state-space equation of the DC-grid shown in Fig. 10 is given by

$$
\left[\begin{array}{c}
\dot{i}_{1} \\
\hdashline \dot{s} \\
\dot{i}_{2} \\
\hdashline \dot{i}_{3}
\end{array}\right]=\left[\begin{array}{c:cc:c}
-\frac{R+d_{1}^{-2} R_{L 1}}{L} & \frac{d_{1}^{-1} d_{2}^{-1}}{K L} & 0 & 0 \\
\hdashline 0 & 0 & 1 & 0 \\
\frac{d_{1}^{-1} d_{2}^{-1} R_{L 1}}{L} & -\frac{2 d_{2}^{-2}}{K L} & -\frac{R}{L} & \frac{d_{2}^{-1} d_{3}^{-1} R_{L 3}}{L} \\
\hdashline 0 & \frac{d_{2}^{-1} \tilde{d}_{3}^{-1}}{K L} & 0 & -\frac{R+2 d_{3}^{-2} R_{L 3}}{L}
\end{array}\right]\left[\begin{array}{c}
i_{1} \\
\hdashline s \\
i_{2} \\
\hdashline i_{3}
\end{array}\right]+\left[\begin{array}{c}
0 \\
0 \\
0 \\
\hdashline \frac{d_{3}^{-1}}{L}
\end{array}\right] E
$$

It follows that the DC-grid can be modeled as an interconnected system of the form $(\mathcal{G} \star \Omega) \Omega_{\mathrm{FF}}$ where

$$
\begin{aligned}
& \mathcal{G}=\operatorname{diag}\left(G_{1}, G_{2}, G_{3}\right), \quad G_{1}:=\left[\begin{array}{c|c}
-\frac{R+d_{1}^{-2} R_{L 1}}{L} & \frac{1}{L} \\
R_{L 1} & 0
\end{array}\right], \\
& G_{2}:=\left[\begin{array}{cc|c|c}
0 & 1 & 0 \\
-\frac{2 d_{2}^{-2}}{K L} & -\frac{R}{L} & \frac{1}{L} \\
\hline \frac{1}{K} & 0 & 0
\end{array}\right], G_{3}:=\left[\begin{array}{cc}
-\frac{R+2 d_{3}^{-2} R_{L 3}}{L} & \frac{1}{L} \\
R_{L 3} & 0
\end{array}\right], \\
& \Omega=\left[\begin{array}{ccc}
0 & d_{1}^{-1} d_{2}^{-1} & 0 \\
d_{1}^{-1} d_{2}^{-1} & 0 & d_{2}^{-1} d_{3}^{-1} \\
0 & d_{2}^{-1} d_{3}^{-1} & 0
\end{array}\right], \Omega_{\mathrm{FF}}=\left[\begin{array}{c}
0 \\
0 \\
d_{3}^{-1}
\end{array}\right] .(44)
\end{aligned}
$$

Note that the interconnected system $(\mathcal{G} \star \Omega) \Omega_{\mathrm{FF}}$ receives the step input $E$. The outputs of the subsystems $G_{1}, G_{2}$, and $G_{3}$ are $v_{1}, v_{2}\left(=K^{-1} s\right)$, and $v_{3}$, respectively. It is clear that $G_{1}, G_{2}$, and $G_{3}$ are all stable, and $G_{1}$ and $G_{3}$ are positive irrespective of $d_{1}$ and $d_{3}$. Moreover, it is easy to confirm that $G_{2}$ is positive (under appropriate state-space realization) if and only if $d_{2} \geq \sqrt{8 L} /(R \sqrt{K})\left(:=d_{2, \min }\right)$. To summarize, we can conclude that the DC-grid in Fig. 10 can be modeled as an interconnected positive system with step input $E$ as long as $d_{2} \geq d_{2, \min }$. Note that $d_{2, \text { min }}$ is small in general since $R \gg L$ in practice. By enforcing positivity, we can specifically guarantee that $s(t) \geq 0(t \geq 0)$ always holds under any reasonable initial conditions.

\section{Computation of Gains $d_{1}, d_{2}$, and $d_{3}$}

Since $\Omega$ and $\Omega_{\mathrm{FF}}$ given by (44) satisfy the condition corresponding to (ii-b) of Corollary 3, we see from (29) of Remark 6 that the design objective (43) is satisfied if

$$
\Psi\left[\begin{array}{ll}
\Omega & \Omega_{\mathrm{FF}}
\end{array}\right]\left[\begin{array}{c}
v_{1}^{\star} \\
K^{-1} s^{\star} \\
v_{3}^{\star} \\
E
\end{array}\right]=\left[\begin{array}{c}
v_{1}^{\star} \\
K^{-1} s^{\star} \\
v_{3}^{\star}
\end{array}\right]
$$

holds where

$$
\begin{aligned}
\Psi & =\operatorname{diag}\left(G_{1}(0), G_{2}(0), G_{3}(0)\right) \\
& =\operatorname{diag}\left(\frac{R_{L 1} d_{1}^{2}}{R d_{1}^{2}+R_{L 1}}, \frac{d_{2}^{2}}{2}, \frac{R_{L 3} d_{3}^{2}}{R d_{3}^{2}+2 R_{L 3}}\right) .
\end{aligned}
$$

From (44) and (46), the condition (45) can be rewritten row by row as

$$
\frac{R_{L 1} d_{1} d_{2}^{-1} K^{-1} s^{\star}}{R d_{1}^{2}+R_{L 1}}=v_{1}^{\star},
$$




$$
\begin{aligned}
& \frac{\left(d_{1}^{-1} v_{1}^{\star}+d_{3}^{-1} v_{3}^{\star}\right)}{2}=d_{2}^{-1} K^{-1} s^{\star}, \\
& \frac{R_{L 3} d_{3}\left(d_{2}^{-1} K^{-1} s^{\star}+E\right)}{R d_{3}^{2}+2 R_{L 3}}=v_{3}^{\star} .
\end{aligned}
$$

The problem to determine $d_{i}(i=1,2,3)$ is essentially nonlinear since $d_{i}$ acts proportionally on $i_{i}$ and reciprocally on $v_{i}(i=1,2,3)$ and hence hard to solve seemingly. This nonlinearity can be seen also from the fact that all the matrices $\Omega, \Omega_{\mathrm{FF}}$, and $\Psi$ depend nonlinearly on $d_{i}(i=1,2,3)$ as clearly shown in (44) and (46). However, the subsystem-based condition (47), which is derived from general positive system theory in Corollary 3, allows us to solve the problem in a straightforward fashion. Namely, once we fix $d_{2}$, then we can determine $d_{1}$ by solving the second order algebraic equation (47a). Here, if (47a) has two nonnegative solutions, we choose smaller one in view of the fact that smaller gain is preferable in practical application. Similarly for $d_{3}$ and (47c). It follows that, for each fixed $d_{2}$, the left-hand side of (47b) can be determined uniquely by (47a) and (47c). Therefore, by plotting the difference of the left and right terms of (47b) for each $d_{2}$, and by finding the value $d_{2}$ on which the difference vanishes, we can obtain $d_{1}, d_{2}$, and $d_{3}$ satisfying (47).

Remark 11: We have derived the synthesis condition (47) by applying (29) given in Remark 6. A merit of such treatment over standard equilibrium analysis is that, by viewing the DC-grid as an interconnected positive system, we can enjoy subsystem-based treatment and derive the compact condition (47) in a constructive fashion. Here, it is of course true that (29) represents the condition to achieve the desired equilibrium for the interconnected positive system $\left(\mathcal{G}_{\mathrm{u}} \star \Omega_{\mathrm{u}}\right) \Omega_{\mathrm{ul}}$ in Fig. 3 . To see this clearly, let us rewrite (29) as in

$$
v_{\mathrm{obj}, \mathrm{u}}=\Psi_{\mathrm{u}}\left(\Omega_{\mathrm{u}} v_{\mathrm{obj}, \mathrm{u}}+\Omega_{\mathrm{ul}} v_{\mathrm{obj}, \mathrm{l}}\right) \text {. }
$$

Then, we see that (29) is a necessary condition to achieve $\lim _{t \rightarrow \infty} \widehat{z}_{\mathrm{u}}(t)=v_{\mathrm{obj}, \mathrm{u}}$ for $\widehat{z}_{1}(t)=v_{\mathrm{obj}, 1}(t \geq 0)$ even for those cases where $\mathcal{G}_{\mathrm{u}}$ is a general (non-positive) system and $\Omega_{\mathrm{u}}$ and $\Omega_{\mathrm{ul}}$ are general matrices (with negative entries). However, in such cases the condition (29) is far from sufficient to achieve the desired equilibrium in general, since we cannot ensure the stability of $\mathcal{G}_{\mathrm{u}} \star \Omega_{\mathrm{u}}$ by relying merely on (29). What is important in Remark 6 is that, if $\mathcal{G}_{\mathrm{u}}$ is positive and $\Omega_{\mathrm{u}}$ and $\Omega_{\mathrm{ul}}$ are nonnegative, the condition (29) ensures the stability of $\mathcal{G}_{\mathrm{u}} \star \Omega_{\mathrm{u}}$ and hence (29) becomes a necessary and sufficient condition to achieve the desired equilibrium. This stability guarantee is the key in Remark 6, even though such stability issue is not necessarily relevant in this particular DCgrid example since we see from physical interpretation that the DC-grid system is stable for any $d_{1}>0, d_{2}>0, d_{3}>0$.

\section{Design Examples}

We let $E=32 \mathrm{~V}, R=1 \Omega, L=1 \mathrm{mH}, K=10 \mathrm{~F}, R_{L 1}=$ $R_{L 3}=20 \Omega$ and consider the two cases for the reference values $v_{1}^{\star}, s^{\star}$, and $v_{3}^{\star}$. Note that $d_{2, \min } \approx 0.0283$ in both cases.

1) Case I: $\left(v_{1}^{\star}, s^{\star}, v_{3}^{\star}\right)=(12 \mathrm{~V}, 50 \mathrm{C}, 24 \mathrm{~V})$ : By the suggested procedure, we computed $\left(d_{1}, d_{2}, d_{3}\right)$ satisfying (47) and obtained $\left(d_{1}, d_{2}, d_{3}\right)=(0.3953,0.1634,0.7785)$.

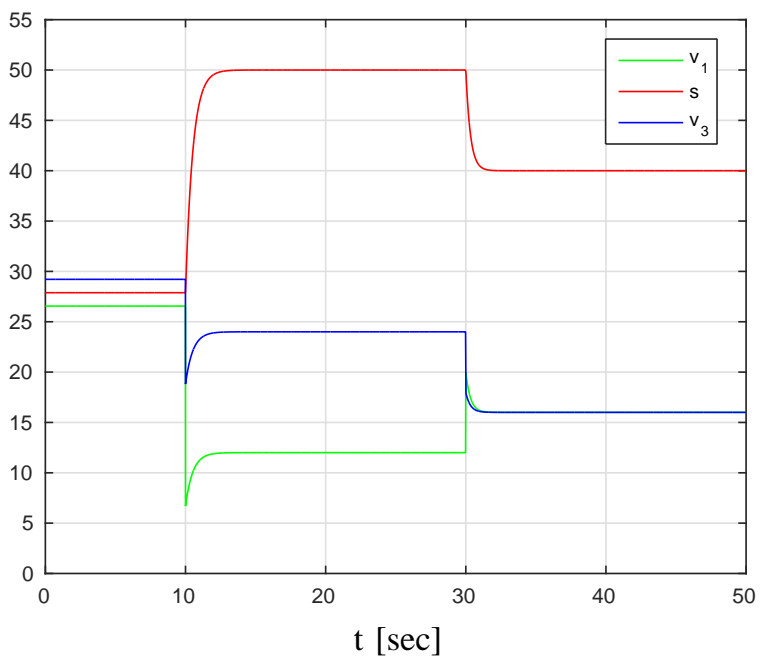

Fig. 11. Simulation results for Cases I and II.

2) Case II: $\left(v_{1}^{\star}, s^{\star}, v_{3}^{\star}\right)=(16 \mathrm{~V}, 40 \mathrm{C}, 16 \mathrm{~V})$ : Again by the suggested procedure, we computed $\left(d_{1}, d_{2}, d_{3}\right)$ satisfying (47) and obtained $\left(d_{1}, d_{2}, d_{3}\right)=(0.5277,0.1301,0.5134)$.

In Fig. 11, we show the simulation results. We assume that the DC-grid is initially at the steady-state with DC-DC converter gains $\left(d_{1}, d_{2}, d_{3}\right)=(1,0.1,1)$ and we switched the gains to the computed values for Case I at $10 \mathrm{sec}$ and for Case II at $30 \mathrm{sec}$. We can confirm that the design objective (43) is successfully achieved.

\section{CONCLUSION}

In this paper, we presented several novel results on the analysis and synthesis of interconnected systems constructed from heterogeneous positive subsystems and a nonnegative interconnection matrix. In particular, we showed that the admissibility, stability, and persistence can be characterized completely in terms of the (weighted) $L_{1}$-induced norm of each positive subsystem and the Frobenius eigenvalue of the interconnection matrix scaled by steady-stage gains of subsystems. We illustrated the usefulness of the analysis results by applying them to formation control of multi-agent systems with positive dynamics and an energy management problem in DC-grids.

It is nonetheless true that the application results are far from complete and positive system theory developed in this paper should be better illustrated in more realistic applications. To find out such convincing applications, we made continuing efforts and partial results have been obtained on positivitybased time-headway control of vehicle platoons [7], [6]. We emphasize that theoretical results in this paper form the basis in this application as well. Still, the application results in [7], [6] remain to be academic, and it is an important future issue to conceive more practical positivity-based control applications to make linear positive system theory most fruitful.

\section{ACKNOWLEDGEMENTS}

This work was supported by JSPS KAKENHI Grant Number 25420436 . 


\section{REFERENCES}

[1] M. Ait Rami and F. Tadeo. Controller synthesis for positive linear systems with bounded controls. IEEE Transactions on Circuits and Systems, 54(2):151-155, 2007.

[2] S. Anand, B. G. Fernandes, and J. M. Guerrero. Distributed control to ensure proportional load sharing and improve voltage regulation in low-voltage DC microgrids. IEEE Transactions on Power Electronics, 28(4):1900-1913, 2013.

[3] F. Blanchini, P. Colaneri, and M. E. Valcher. Co-positive Lyapunov functions for the stabilization of positive switched systems. IEEE Transactions on Automatic Control, 57(12):3038-3050, 2012.

[4] S. Boyd and V. Balakrishnan. Convex Optimization. Cambridge University Press, Cambridge, 2004.

[5] C. Briat. Robust stability and stabilization of uncertain linear positive systems via integral linear constraints: $L_{1}$-gain and $L_{\infty}$-gain characterization. International Journal of Robust and Nonlinear Control, 23(17):1932-1954, 2013.

[6] Y. Ebihara. Analysis and synthesis of delay interconnected positive systems with external inputs and formation control of moving objects. In Proc. Conference on Decision and Control, pages 6367-6372, 2015.

[7] Y. Ebihara, T. Matsumura, T. Hagiwara, D. Peaucelle, and D. Arzelier. Analysis and synthesis of interconnected positive systems with external inputs. In Proc. of the 8th IFAC Robust Control Design, pages 160-165, 2015.

[8] Y. Ebihara, D. Peaucelle, and D. Arzelier. $L_{1}$ gain analysis of linear positive systems and its application. In Proc. Conference on Decision and Control, pages 4029-4034, 2011.

[9] Y. Ebihara, D. Peaucelle, and D. Arzelier. Analysis and synthesis of interconnected SISO positive systems with switching. In Proc. Conference on Decision and Control, pages 6372-6378, 2013.

[10] Y. Ebihara, D. Peaucelle, and D. Arzelier. Stability and persistence analysis of large scale interconnected positive systems. In Proc. European Control Conference, pages 3366-3371, 2013.

[11] Y. Ebihara, D. Peaucelle, and D. Arzelier. Efficient convergence rate analysis of multi-agent positive systems under formation control. In Proc. the 19th IFAC World Congress, pages 3790-3796, 2014.

[12] L. Farina and S. Rinaldi. Positive Linear Systems: Theory and Applications. John Wiley and Sons, Inc., 2000.

[13] N. Fujimori, L. Liu, S. Hara, and D. Tsubakino. Hierarchical network synthesis for output consensus by eigenvector-based interlayer connections. In Proc. Conference on Decision and Control, pages 1449-1454, 2011.

[14] M. Fujita and T. Hatanaka. Cooperative control: Basic results on consensus and coverage control problems (in japanese). In Proc. the 52nd Annual Conference of the ISCIE, pages 1-6, 2008.

[15] M. Green and D. Limebeer. Linear Robust Control. Prentice-Hall, 1995.

[16] L. Gurvits, R. Shorten, and O. Mason. On the stability of switched positive linear systems. IEEE Transactions on Automatic Control, 52(6):1099-1103, 2007.

[17] W. M. Haddad and V. Chellaboina. Stability theory for nonnegative and compartmental dynamical systems with time delay. Systems and Control Letters, 51(5):355-361, 2004.

[18] S. Hara, T. Hayakawa, and H. Sugata. Stability analysis of linear systems with generalized frequency variables and its application to formation control. In Proc. Conference on Decision and Control, pages 14591466, 2007.

[19] R. A. Horn and C. A. Johnson. Matrix Analysis. Cambridge University Press, New York, 1985.

[20] T. Iwasaki and G. Shibata. LPV system analysis via quadratic separator for uncertain implicit systems. IEEE Transactions on Automatic Control, 46(8):1195-1208, 2001.

[21] T. Kaczorek. Positive $1 D$ and 2D Systems. Springer, London, 2001.

[22] H. Kakigano, Y. Miura, and T. Ise. Distributed control to ensure proportional load sharing and improve voltage regulation in low-voltage DC microgrids. IEEE Transactions on Power Electronics, 28(5):2246$2258,2013$.

[23] J. H. Kim and T. Hagiwara. Quasi-finite-rank approximation of compression operators on $l_{\infty}[0, h)$ with application to stability analysis of time-delay systems. IET Control Theory and Applications, 8(2):77-85, 2014.

[24] K. Konishi, Y. Sugitani, and N. Hara. Dynamics of DC bus networks and their stabilization by decentralized delayed feedback. Physical Review E, 91(1):012911, 2015.

[25] P. Li and J. Lam. Positive state-bounding observer for positive interval continuous-time systems with time delay. International Journal of Robust and Nonlinear Control, 22(11):1244-1257, 2011.
[26] O. Mason and R. Shorten. On linear copositive Lyapunov function and the stability of switched positive linear systems. IEEE Transactions on Automatic Control, 52(7):1346-1349, 2007.

[27] R. Olfati-Saber, J. A. Fax, and R. M. Murray. Consensus and cooperation in networked multi-agent systems. Proceedings of the IEEE, 95(1):215233, 2007.

[28] R. Olfati-Saber and R. M. Murray. Consensus problems in networks of agents with switching topology and time-delays. IEEE Transactions on Automatic Control, 49(9):1520-1533, 2004.

[29] D. Peaucelle, D. Arzelier, D. Henrion, and F. Gouaisbaut. Quadratic separation for feedback connection of an uncertain matrix and an implicit linear transformation. Automatica, 43(5):795-804, 2007.

[30] I. Polushin, H. J. Marquez, A. Tayebi, and P. X. Liu. A multichannel IOS small gain theorem for systems with multiple time-varying communication delays. IEEE Transactions on Circuits and Systems, 54(2):404-409, 2009.

[31] A. Rantzer. Distributed control of positive systems. In Proc. Conference on Decision and Control, pages 6608-6611, 2011.

[32] A. Rantzer. On the Kalman-Yakubovich-Popov lemma for positive systems. In Proc. Conference on Decision and Control, pages 74827484, 2012.

[33] B. S. Rüffera, C. M. Kellett, and S. R. Weller. Connection between cooperative positive systems and integral input-to-state stability of largescale systems. Automatica, 46:1019-1027, 2010.

[34] C. W. Scherer. LPV control and full block multipliers. Automatica, 37(3):361-375, 2001.

[35] R. Shorten, O. Mason, and K. Wulff. Convex Cones, Lyapunov Functions, and the Stability of Switched Linear Systems, volume 3335 of Lecture Notes in Computer Science. Springer-Verlag, Berlin, 2005.

[36] K. Takaba. A study on distributed cooperative control of large-scale plants (in japanese). In Proc. The 2nd Multi-symposium on Control Systems, pages 532-2, 2015.

[37] T. Tanaka and C. Langbort. The bounded real lemma for internally positive systems and $H_{\infty}$ structured static state feedback. IEEE Transactions on Automatic Control, 56(9):2218-2223, 2011.

[38] H. G. Tanner, A. Jadbabaie, and G. J. Pappas. Stable flocking of mobile agents, part I: Fixed topology. In Proc. Conference on Decision and Control, pages 2010-2015, 2003.

[39] H. G. Tanner, A. Jadbabaie, and G. J. Pappas. Stable flocking of mobile agents, part II: Dynamic topology. In Proc. Conference on Decision and Control, pages 2016-2021, 2003.

[40] M. E. Valcher and P. Misra. On the stabilizability and consensus of positive homogeneous multi-agent dynamical systems. IEEE Transactions on Automatic Control, 59(7):1936-1941, 2014.

[41] C. W. Wu and L. O. Chua. Synchronization in an array of linearly coupled dynamical systems. IEEE Trans. Circuit Syst.-I, 42(8):430 447, 1995.

[42] C. W. Wu and L. O. Chua. On a conjecture regarding the synchronization in an array of linearly coupled dynamical systems. IEEE Trans. Circuit Syst. $-I$, 43(2):161-165, 1996.

[43] A. Zappavigna, P. Colaneri, J. C. Geromel, and R. Shorten. Dwell time analysis for continuous-time switched linear positive systems. In Proc. American Control Conference, pages 6256-6261, 2010.

[44] A. Zappavigna, P. Colaneri, J. C. Geromel, and R. Shorten. Stabilization of continuous-time switched linear positive systems. In Proc. American Control Conference, pages 3275-3280, 2010.

\section{APPENDICES}

\section{A. Proof of Lemma 2}

Proof of Lemma 2: We will prove the equivalence of (i) and (ii). The equivalence of (i) and (iii) follows similarly.

(i) $\Rightarrow$ (ii) Suppose (i) holds. Then, from (iii) of Lemma 1, there exist $h_{1} \in \mathbb{R}_{++}^{n_{1}}$ and $h_{2} \in \mathbb{R}_{++}^{n_{2}}$ such that

$$
h_{1}^{T} P+h_{2}^{T} R<0, \quad h_{1}^{T} Q+h_{2}^{T} S<0 .
$$

The first inequality implies $h_{1}^{T} P<-h_{2}^{T} R \leq 0$ since $h_{2} \in \mathbb{R}_{++}^{n_{2}}$ and $R \in \mathbb{R}_{+}^{n_{2} \times n_{1}}$. Hence, due to (iii) in Lemma 1 , $P$ is Hurwitz stable and due to (ii) in Lemma 1 we have $P^{-1} \leq 0$. The first inequality in (48) therefore implies $h_{1}^{T}>-h_{2}^{T} R P^{-1}$. By combining this inequality to the second inequality and noting that $Q \in \mathbb{R}_{+}^{n_{1} \times n_{2}}$, we have 


$$
h_{2}^{T}\left(S-R P^{-1} Q\right)<0 .
$$

It is obvious that $S-R P^{-1} Q$ is Metzler since $P^{-1} \leq 0$ and hence, again from (iii) of Lemma 1, we conclude that $S-R P^{-1} Q$ is Hurwitz stable.

(ii) $\Rightarrow$ (i) Suppose (ii) holds. Then, from (iii) of Lemma 1, there exists $h_{2} \in \mathbb{R}_{++}^{n_{2}}$ such that (49) holds. It follows that there exists $\varepsilon>0$ such that $h_{2}^{T} S-\left(h_{2}^{T} R+\varepsilon \mathbf{1}^{n_{1} T}\right) P^{-1} Q<0$ where $1^{n_{1}} \in \mathbb{R}^{n_{1}}$ stands for the all-ones vector. If we define $h_{1}:=-\left(\left(h_{2}^{T} R+\varepsilon \mathbf{1}^{n_{1} T}\right) P^{-1}\right)^{T}$, we have $h_{1} \in \mathbb{R}_{++}^{n_{1}}$ since $P$ is Hurwitz and hence $P^{-1} \leq 0$. In addition, we readily obtain

$$
h_{1}^{T} Q+h_{2}^{T} S<0, \quad h_{1}^{T} P+h_{2}^{T} R=-\varepsilon \mathbf{1}^{n_{1} T}<0 .
$$

Again, from (iii) of Lemma 1, this shows that the Metzler matrix $\Pi$ in (i) is Hurwitz stable.

\section{B. Proof of Theorem 1}

Proof of Theorem 1: We prove (ii) $\Rightarrow$ (i), (i) $\Rightarrow$ (ii), (ii) $\Rightarrow$ (iii) and (iii) $\Rightarrow$ (ii) in order.

(ii) $\Rightarrow$ (i) Suppose (ii) holds for some $h>0$. Then $A \in \mathbb{M}^{n \times n}$ is obviously Hurwitz from (iii) of Lemma 1 . In addition, there exists $\varepsilon>0$ such that

$$
\left[\begin{array}{ll}
h^{T} A+q_{z}^{T} C & \left.h^{T} B+q_{z}^{T} D-(\gamma-\varepsilon) q_{w}^{T}\right]<0 .
\end{array}\right.
$$

It follows that, for any $x \in \mathbb{R}^{n}$ and $w \in \mathbb{R}^{n_{w}}$ satisfying $\left[x^{T} w^{T}\right]^{T} \geq 0$, we have

$$
\left[h^{T} A+q_{z}^{T} C h^{T} B+q_{z}^{T} D-(\gamma-\varepsilon) q_{w}^{T}\right]\left[\begin{array}{l}
x \\
w
\end{array}\right] \leq 0 .
$$

This can be rearranged as

$$
h^{T}(A x+B w)+q_{z}^{T}(C x+D w)-(\gamma-\varepsilon) q_{w}^{T} w \leq 0 .
$$

Since $G$ is positive, we note that $x(t) \geq 0 \forall t \in[0, \infty)$ holds for any input signal $w \in L_{1+}^{n_{w}}$ and $x(0)=0$. From this fact and the above inequality, we see that along the trajectory of the system $G$ the following relation holds:

$$
\begin{array}{r}
h^{T} \dot{x}(t)+q_{z}^{T} z(t)-(\gamma-\varepsilon) q_{w}^{T} w(t) \leq 0 \\
\forall t \in[0, \infty) \quad \forall w \in L_{1+}^{n_{w}} .
\end{array}
$$

By integrating the above inequality over $[0, T]$, we have

By noting that $h^{T} x(T) \geq 0$, it is obvious that

$$
\int_{0}^{T} q_{z}^{T} z(t) d t-(\gamma-\varepsilon) \int_{0}^{T} q_{w}^{T} w(t) d t \leq 0 \quad \forall w \in L_{1+}^{n_{w}} .
$$

Moreover, by restricting $w$ to be such that $\left\|q_{w}^{T} w\right\|_{1}=1$ and letting $T \rightarrow \infty$, we see that $\int_{0}^{\infty} q_{z}^{T} z(t) d t-(\gamma-\varepsilon) \leq 0$ holds for all $w \in L_{1+}^{n_{w}}$ such that $\left\|q_{w}^{T} w\right\|_{1}=1$. It follows that (i) is satisfied.

(i) $\Rightarrow$ (ii) To prove the assertion by contradiction, suppose (ii) does not hold for any $h>0$. Then only the following two cases are possible:

(a) $A$ is not Hurwitz stable.

(b) $A$ is Hurwitz stable but (5) does not hold for any $h>0$.
Since (a) clearly contradicts (i), we only consider the case (b). Then, from the strong alternative for linear inequalities [4, Section 5.8], there exist $g_{1} \in \mathbb{R}_{+}^{n}$ and $g_{2} \in \mathbb{R}_{+}^{n_{w}}$, not simultaneously zero, such that

$$
A g_{1}+B g_{2} \geq 0, \quad q_{z}^{T} C g_{1}+\left(q_{z}^{T} D-\gamma q_{w}^{T}\right) g_{2} \geq 0 .
$$

If $g_{2}=0$, we have $g_{1} \neq 0, g_{1} \geq 0$, and $A g_{1} \geq 0$, which contradicts the Hurwitz stability of $A$ (see (iv) of Lemma 1). Therefore it suffices to consider the case where $A$ is Hurwitz stable and $g_{2} \neq 0$. With this in mind, let us note that the first inequality above implies $g_{1} \leq-A^{-1} B g_{2}$ since $A^{-1} \leq 0$ from (ii) of Lemma 1. By substituting this into the second inequality, we obtain $\left(q_{z}^{T} G(0)-\gamma q_{w}^{T}\right) g_{2} \geq 0$. Moreover, since $g_{2} \geq 0$ and $g_{2} \neq 0$ as noted above, the following inequality must hold for at least one index $j^{\star}\left(1 \leq j^{\star} \leq n_{w}\right)$ :

$$
\left(q_{z}^{T} G(0)\right)_{j^{\star}}-\gamma q_{w, j^{\star}} \geq 0 .
$$

In the following, we assume $q_{w, j^{\star}}=1$ without loss of generality. For a given $T>0$, we also define a linear operator $\mathbb{I}_{T}$ as follows:

$$
\mathbb{I}_{T} \zeta:=\left\{\begin{array}{cl}
\zeta(t) & (0 \leq t \leq T) \\
0 & (T<t)
\end{array} .\right.
$$

Now we move on to the final stage of the proof. To this end, let us define a constant input signal $w_{\text {st }}(t):=e_{j^{\star}} \in \mathbb{R}_{+}^{n_{w}}$, where $e_{i}$ is the $i$-th standard basis of $\mathbb{R}^{n_{w}}$. We also denote by $z_{\text {st }}(t)$ the response of the system $G$ to the input $w_{\text {st }}(t)$. Then, since $\lim _{t \rightarrow \infty} z_{\mathrm{st}}(t)=G(0) e_{j^{\star}}$, we see that for any $\varepsilon>0$ satisfying $\gamma-\varepsilon>0$, there exists $T_{\varepsilon}>0$ such that

$$
q_{z}^{T} z_{\mathrm{st}}(t)-q_{z}^{T} G(0) e_{j^{\star}}>-\frac{\varepsilon}{2} \quad \forall t>T_{\varepsilon} .
$$

From (52), this implies

$$
q_{z}^{T} z_{\mathrm{st}}(t)>\gamma-\frac{\varepsilon}{2}>0 \quad \forall t>T_{\varepsilon} .
$$

If we define another input signal $w_{T}^{\star}(t):=\mathbb{I}_{T} w_{\text {st }}$ for a given $T\left(>T_{\varepsilon}\right)$ and denote by $z_{T}^{\star}(t)$ the corresponding output signal, then we have $\left\|q_{w}^{T} w_{T}^{\star}\right\|_{1}=T, z_{T}^{\star}(t)=z_{\text {st }}(t)(0 \leq t \leq T)$ and hence

$$
\begin{aligned}
& \frac{\left\|q_{z}^{T} z_{T}^{\star}\right\|_{1}}{\left\|q_{w}^{T} w_{T}^{\star}\right\|_{1}} \\
& =\frac{1}{T}\left(\int_{0}^{T_{\varepsilon}} q_{z}^{T} z_{T}^{\star}(t) d t+\int_{T_{\varepsilon}}^{T} q_{z}^{T} z_{T}^{\star}(t) d t+\int_{T}^{\infty} q_{z}^{T} z_{T}^{\star}(t) d t\right) \\
& \geq \frac{1}{T} \int_{T_{\varepsilon}}^{T} q_{z}^{T} z_{T}^{\star}(t) d t \geq \frac{\left(\gamma-\frac{\varepsilon}{2}\right)\left(T-T_{\varepsilon}\right)}{T} \\
& =\gamma-\frac{\varepsilon}{2}-\left(\gamma-\frac{\varepsilon}{2}\right) \frac{T_{\varepsilon}}{T} .
\end{aligned}
$$

Therefore, for the particular choice of

$$
T>\frac{\gamma-\frac{\varepsilon}{2}}{\frac{\varepsilon}{2}} T_{\varepsilon}=\frac{2 \gamma-\varepsilon}{\varepsilon} T_{\varepsilon}\left(>T_{\varepsilon}\right),
$$

we have $\left\|q_{z}^{T} z_{T}^{\star}\right\|_{1} /\left\|q_{w}^{T} w_{T}^{\star}\right\|_{1}>\gamma-\varepsilon$. Since $\varepsilon>0$ can be taken arbitrarily small, this implies $\left\|G_{q_{z}, q_{w}}\right\|_{1+} \geq \gamma$, which contradicts (i).

$\underline{\text { (ii) } \Rightarrow \text { (iii) }}$ The linear inequality (5) implies $A \in \mathbb{H}^{n \times n}$ and

$$
h^{T}>-q_{z}^{T} C A^{-1}, \quad h^{T} B+q_{z}^{T} D<\gamma q_{w}^{T}
$$


since we have $A^{-1} \leq 0$ from (ii) of Lemma 1 . By substituting the former into the latter, we obtain (6).

(iii) $\Rightarrow$ (ii) Let us fix $v \in \mathbb{R}_{++}^{n}$ such that $v^{T} A<0$. Then, the condition (6) implies that there exists $\varepsilon>0$ such that

$$
q_{z}^{T} D+\left(-q_{z}^{T} C A^{-1}+\varepsilon v^{T}\right) B<\gamma q_{w}^{T} .
$$

If we define $h:=\left(-q_{z}^{T} C A^{-1}+\varepsilon v^{T}\right)^{T}>0$, we readily obtain

$$
h^{T} A+q_{z}^{T} C=\varepsilon v^{T} A<0, \quad h^{T} B+q_{z}^{T} D-\gamma q_{w}^{T}<0 .
$$

This clearly shows that (5) holds.

\section{Proof of Theorem 2}

Proof of Theorem 2: For each subsystem, let us define

$$
\begin{aligned}
B_{i} & :=\left[\begin{array}{ccccccc}
B_{i, 1} & \cdots & B_{i, i-1} & B_{i, i+1} & \cdots & B_{i, N}
\end{array}\right] \\
C_{i}:= & {\left[\begin{array}{c}
C_{1, i} \\
\vdots \\
C_{i-1, i} \\
C_{i+1, i} \\
\vdots \\
C_{N, i}
\end{array}\right], D_{i}:=\left[\begin{array}{cccccc}
D_{1, i, 1} & \cdots & D_{1, i, i-1} & D_{1, i, i+1} & \cdots & D_{1, i, N} \\
\vdots & & \vdots & \vdots & & \vdots \\
D_{i-1, i, 1} & \cdots & D_{i-1, i, i-1} & D_{i-1, i, i+1} & \cdots & D_{i-1, i, N} \\
D_{i+1, i, 1} & \cdots & D_{i+1, i, i-1} & D_{i+1, i, i+1} & \cdots & D_{i+i, i, N} \\
\vdots & & \vdots & \vdots & & \vdots \\
D_{N, i, 1} & \cdots & D_{N, i, i-1} & D_{N, i, i+1} & \cdots & D_{N, i, N}
\end{array}\right] }
\end{aligned}
$$

and $z_{i}, w_{i}(i=1, \cdots, N)$ by (18). Then, the system $\mathcal{G}$ can be written in the form of (10) with (11) and (12). Therefore the interconnection of subsystems $G_{i}$ with (16) can be seen as an interconnection with $\mathcal{G}$ and a matrix $\Omega$ precisely given in the following. From (16) and (18), we see that the interconnection matrix $\Omega$ of this case is nothing but a permutation matrix that permutes $z_{i j}$ and $z_{j i}$ in $\widehat{z}$, i.e.,

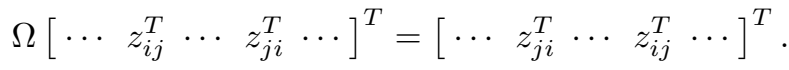

A concrete example of $\Omega$ is given in (19). Since $\Omega$ is a permutation matrix, we see that $\Omega \geq 0$. It follows from Lemma 3 that the interconnected system is admissible and stable if and only if the Metzler matrix

$$
\left[\begin{array}{cc}
\mathcal{A} & \mathcal{B} \Omega \\
\mathcal{C} & \mathcal{D} \Omega-I
\end{array}\right]
$$

is Hurwitz stable. This can be restated equivalently that there exists $h_{i} \in \mathbb{R}_{++}^{n_{i}}$ and $q_{i j} \in \mathbb{R}_{++}^{n_{w_{i j}}}(i, j=1, \cdots, N, i \neq j)$ such that

$$
\begin{aligned}
& {\left[\begin{array}{c}
\widehat{h} \\
\widehat{q}_{z}
\end{array}\right]^{T}\left[\begin{array}{cc}
\mathcal{A} & \mathcal{B} \Omega \\
\mathcal{C} & \mathcal{D} \Omega-I
\end{array}\right]<0,} \\
& \widehat{h}:=\left[\begin{array}{lll}
h_{1}^{T} & \cdots & h_{N}^{T}
\end{array}\right]^{T}, \widehat{q}_{z}:=\left[\begin{array}{lll}
q_{z, 1}^{T} & \cdots & q_{z, N}^{T}
\end{array}\right]^{T} .
\end{aligned}
$$

Here, $q_{z, i}(i=1, \cdots, N)$ are given by (17). Since $\Omega$ is a permutation matrix, we see that (54) holds if and only if

$$
\left[\begin{array}{c}
\widehat{h}^{T} \\
\widehat{q}_{z}
\end{array}\right]^{T}\left[\begin{array}{cc}
\mathcal{A} & \mathcal{B} \\
\mathcal{C} & \mathcal{D}-\Omega^{T}
\end{array}\right]<0
$$

Moreover, we see from the property represented by (53) that

$$
\widehat{q}_{z}^{T} \Omega^{T}=\left(\Omega \widehat{q}_{z}\right)^{T}=\widehat{q}_{w}^{T}, \quad \widehat{q}_{w}:=\left[\begin{array}{lll}
q_{w, 1}^{T} & \cdots & q_{w, N}^{T}
\end{array}\right]^{T} .
$$

Here, $q_{w, i}(i=1, \cdots, N)$ are given by (17). It follows that (55) can be divided into $N$ inequalities as in

$$
\begin{array}{r}
\left.h_{i}^{T} A_{i}+q_{z, i}^{T} C_{i} \quad h_{i}^{T} B_{i}+q_{z, i}^{T} D_{i}-q_{w, i}^{T}\right]<0 \\
(i=1, \cdots, N) .
\end{array}
$$

From Theorem 1, (56) holds if and only if $\left\|G_{i, q_{z, i}, q_{w, i}}\right\|_{1+}<$ $1(i=1, \cdots, N)$. This completes the proof.

\section{Proof of Lemma 4}

Proof of Lemma 4: We give the proof for the controllability only. The result for the observability readily follows from the system duality. For contradiction, suppose $v:=A^{-1} B<0$ does not hold. From the underlying assumptions $A \in\left\{\mathbb{M}^{n \times n} \cap\right.$ $\left.\mathbb{H}^{n \times n}\right\}$ and $B \in \mathbb{R}_{+}^{n}$, we see that $v \leq 0$ definitely holds since $A^{-1} \leq 0$. Therefore there exists a nonempty index set $\mathcal{I} \subset$ $\{1, \cdots, n\}$ such that $v_{i}=0(i \in \mathcal{I}), v_{i}<0\left(i \in \mathcal{I}^{\mathrm{c}}\right)$ where $\mathcal{I}^{\mathrm{c}}$ is the complement of $\mathcal{I}$. Again from $B(=A v) \in \mathbb{R}_{+}^{n}$, it follows that

$$
A v \geq 0, \quad v_{i}=0(i \in \mathcal{I}), \quad v_{i}<0\left(i \in \mathcal{I}^{\mathrm{c}}\right) .
$$

Since $A \in \mathbb{M}^{n \times n}$, the above conditions imply $A_{i j}=0(i \in$ $\left.\mathcal{I}, j \in \mathcal{I}^{\mathrm{c}}\right)$. Therefore we have $(A v)_{i}=0(i \in \mathcal{I})$. Repeating the same argument, we obtain $\left(A^{k} v\right)_{i}=0(i \in \mathcal{I}, k=$ $0,1, \cdots, n-1)$. Then, if we denote by $U_{\mathrm{c}}$ the controllability matrix for the pair $(A, B)$, we have

$$
\begin{aligned}
\operatorname{rank}\left(U_{\mathrm{c}}\right) & =\operatorname{rank}\left(\left[\begin{array}{llll}
B & A B & \cdots & A^{n-1} B
\end{array}\right]\right) \\
& =\operatorname{rank}\left(\left[\begin{array}{llll}
A v & A^{2} & \cdots & A^{n} v
\end{array}\right]\right) \\
& =\operatorname{rank}\left(\left[\begin{array}{llll}
v & A v & \cdots & A^{n-1} v
\end{array}\right]\right) \\
& \leq n-|\mathcal{I}|
\end{aligned}
$$

where $|\mathcal{I}|$ is the cardinality of $\mathcal{I}$. This implies that $(A, B)$ is not controllable and hence the proof is completed.

\section{E. Proof of $(I V)$ in Theorem 5}

For the proof, we need the next lemma.

Lemma 5: For given $A \in \mathbb{M}^{n \times n}, B \in \mathbb{R}_{+}^{n \times 1}$, and $C \in \mathbb{R}_{+}^{1 \times n}$, suppose $(A, B)$ is controllable and $(A, C)$ is observable. Then, for a given $\alpha \in \mathbb{R}$ such that $\alpha I+A \in \mathbb{R}_{+}^{n \times n}$, we have $C(\alpha I+$ $A)^{i} B>0$ for at least one index $i \in\{0, \cdots, n-1\}$.

Proof of Lemma 5: Since $(A, B)$ is controllable and $(A, C)$ is observable, $(\alpha I+A, B)$ is controllable and $(\alpha I+A, C)$ is observable. Therefore we see that $U:=U_{\mathrm{o}, \alpha} U_{\mathrm{c}, \alpha} \in \mathbb{R}_{+}^{n \times n}$ is nonsingular where $U_{\mathrm{c}, \alpha}$ and $U_{\mathrm{o}, \alpha}$ stand for the controllability and observability matrices for the pairs $(\alpha I+A, B)$ and $(\alpha I+A, C)$, respectively. The first row of $U$ given by $\left[C B C(\alpha I+A) B \cdots C(\alpha I+A)^{n-1} B\right]$ is nonzero since $U$ is nonsingular from the controllability and observability assumption. Therefore the assertion readily follows.

Proof of (IV) in Theorem 5: Let us define $\Omega_{\mathcal{D}}:=\Omega(I-$ $\mathcal{D} \Omega)^{-1}$ as in the proof of (III). Then, from the assertion (I) already validated, the matrix $\mathcal{D} \Omega-I$ is Hurwitz and hence $\rho(\mathcal{D} \Omega)=\lambda_{\mathrm{F}}(\mathcal{D} \Omega)<1$. It follows that

$$
\Omega_{\mathcal{D}}=\Omega(I-\mathcal{D} \Omega)^{-1}=\Omega \sum_{i=0}^{\infty}(\mathcal{D} \Omega)^{i} \geq \Omega \geq 0 .
$$

Since $\Omega$ is irreducible from (iii), the above inequality implies $\Omega_{\mathcal{D}}$ is also irreducible and $\Omega_{\mathcal{D}} \in \mathbb{R}_{+}^{N \times N}$.

With this in mind, suppose $\mathcal{A}_{\mathrm{cl}}$ is reducible for contradiction. Then, for $\alpha>0$ such that $\alpha I+\mathcal{A} \geq 0$, there exists a permutation matrix $P$ such that

$$
\begin{aligned}
& P^{T}\left(\alpha I+\mathcal{A}+\mathcal{B} \Omega_{\mathcal{D}} \mathcal{C}\right) P \in \mathbb{W}_{+}, \\
& \mathbb{W}:=\left\{W=\left[\begin{array}{cc}
Q & R \\
0_{n_{\widehat{x}}-r, r} & S
\end{array}\right]: Q \in \mathbb{R}^{r \times r},\right. \\
& \left.\quad S \in \mathbb{R}^{\left(n_{\widehat{x}}-r\right) \times\left(n_{\widehat{x}}-r\right)}, 1 \leq r \leq n_{\widehat{x}}-1\right\}, \\
& \mathbb{W}_{+}:=\mathbb{W} \cap \mathbb{R}_{+}^{n_{\widehat{x}} \times n_{\widehat{x}}} .
\end{aligned}
$$


Since $\alpha I+\mathcal{A}$ and $\mathcal{B} \Omega_{\mathcal{D}} \mathcal{C}$ are both nonnegative, the above condition implies

$$
\mathcal{Y}_{\mathcal{A}}:=P^{T}(\alpha I+\mathcal{A}) P \in \mathbb{W}_{+}, \mathcal{Y}_{\mathcal{B C}}:=P^{T} \mathcal{B} \Omega_{\mathcal{D}} \mathcal{C} P \in \mathbb{W}_{+} .
$$

To proceed, let us define

$$
n_{\max }:=\max _{i=1, \cdots, N} n_{i}, U:=\sum_{i=0}^{n_{\max }-1}\left(P^{T}(\alpha I+\mathcal{A}) P\right)^{i} \in \mathbb{W}_{+} .
$$

Then, from Lemma 5 , we have $\mathcal{X}:=\mathcal{C} P U P^{T} \mathcal{B} \in \mathbb{D}_{++}^{N \times N}$. With the matrix $U \in \mathbb{W}_{+}$defined above, we also have

$$
\begin{aligned}
& \mathcal{Y}_{\mathcal{B C}}=P^{T} \mathcal{B} \Omega_{\mathcal{D}} \mathcal{C} P \in \mathbb{W}_{+}, \\
& \mathcal{Y}_{\mathcal{B C}} U \mathcal{Y}_{\mathcal{B C}}=P^{T} \mathcal{B} \Omega_{\mathcal{D}} \mathcal{X} \Omega_{\mathcal{D}} \mathcal{C} P \in \mathbb{W}_{+}, \\
& \mathcal{Y}_{\mathcal{B C}}\left(U \mathcal{Y}_{\mathcal{B C}}\right)^{2}=P^{T} \Omega_{\mathcal{D}}\left(\mathcal{X} \Omega_{\mathcal{D}}\right)^{2} \mathcal{C} P \in \mathbb{W}_{+}, \\
& \quad \vdots \\
& \mathcal{Y}_{\mathcal{B C}}\left(U \mathcal{Y}_{\mathcal{B C}}\right)^{N-1}=P^{T} \mathcal{B}_{\mathcal{D}}\left(\mathcal{X} \Omega_{\mathcal{D}}\right)^{N-1} \mathcal{C} P \in \mathbb{W}_{+} .
\end{aligned}
$$

It follows that

$$
P^{T} \mathcal{B Z C P} \in \mathbb{W}_{+}, \quad \mathcal{Z}:=\Omega_{\mathcal{D}} \sum_{i=0}^{N-1}\left(\mathcal{X} \Omega_{\mathcal{D}}\right)^{i} .
$$

Since $\Omega_{\mathcal{D}}$ is irreducible and $\mathcal{X} \in \mathbb{D}_{++}^{N \times N}$, it is obvious that $\mathcal{X} \Omega_{\mathcal{D}}$ is irreducible. Moreover, since $\mathcal{X} \Omega_{\mathcal{D}} \in \mathbb{R}_{+}^{N \times N}$, we see from (b) of Proposition 2 that $\sum_{i=0}^{N-1}\left(\mathcal{X} \Omega_{\mathcal{D}}\right)^{i} \in \mathbb{R}_{++}^{N \times N}$. Since $\Omega_{\mathcal{D}}$ is irreducible and $\Omega_{\mathcal{D}} \in \mathbb{R}_{+}^{N \times N}$, this further indicates that $\mathcal{Z} \in \mathbb{R}_{++}^{N \times N}$.

Now we move onto the final stage of the proof. To this end, let us define $\mathcal{A}_{P}:=P^{T} \mathcal{A} P, \mathcal{B}_{P}:=P^{T} \mathcal{B}, \mathcal{C}_{P}:=\mathcal{C} P$ and partition $\mathcal{B}_{P}$ and $\mathcal{C}_{P}$ as follows:

$$
\begin{aligned}
& \mathcal{B}_{P}=:\left[\begin{array}{c}
\mathcal{B}_{P, 1} \\
\mathcal{B}_{P, 2}
\end{array}\right], \mathcal{B}_{P, 1} \in \mathbb{R}_{+}^{r \times N}, \mathcal{B}_{P, 2} \in \mathbb{R}_{+}^{\left(n_{\widehat{x}}-r\right) \times N}, \\
& \mathcal{C}_{P}=:\left[\mathcal{C}_{P, 1} \mathcal{C}_{P, 2}\right], \mathcal{C}_{P, 1} \in \mathbb{R}_{+}^{N \times r}, \mathcal{C}_{P, 2} \in \mathbb{R}_{+}^{N \times\left(n_{\widehat{x}}-r\right)} .
\end{aligned}
$$

Then, from (58) and (59), we have

$$
\begin{aligned}
& \mathcal{A}_{P} \in \mathbb{W}, \\
& \mathcal{B}_{P} \mathcal{Z} \mathcal{C}_{P} \in \mathbb{W}_{+}, \quad \mathcal{Z} \in \mathbb{R}_{++}^{N \times N} .
\end{aligned}
$$

Here, in relation to (61), suppose $\mathcal{B}_{P, 2} \neq 0$. Then, from (61), we have $\mathcal{C}_{P, 1}=0$. On the other hand, suppose $\mathcal{C}_{P, 1} \neq 0$. Then, again from (61), we have $\mathcal{B}_{P, 2}=0$. It follows that $\mathcal{B}_{P, 2}=0$ or $\mathcal{C}_{P, 1}=0$ holds. From the form of $\mathcal{A}_{P}$ given by (60), the former case implies $\left(\mathcal{A}_{P}, \mathcal{B}_{P}\right)$ is not controllable, and the latter case implies $\left(\mathcal{A}_{P}, \mathcal{C}_{P}\right)$ is not observable. This contradicts to the assumption that $\left(A_{i}, B_{i}\right)$ is controllable and $\left(A_{i}, C_{i}\right)$ is observable for $i=1, \cdots, N$ (and hence $(\mathcal{A}, \mathcal{B})$ and $(\mathcal{A}, \mathcal{C})$ are controllable and observable, respectively). This completes the proof.

\section{F. Proof of Corollary 3}

Proof of Corollary 3: The state-space equation of $\left(\mathcal{G}_{\mathrm{u}} \star\right.$ $\left.\Omega_{\mathrm{u}}\right) \Omega_{\mathrm{ul}}$ is given by

$$
\begin{aligned}
& \left\{\begin{array}{l}
\dot{\widehat{x}}_{\mathrm{u}}=\mathcal{A}_{\mathrm{cl}, \mathrm{u}} \widehat{x}_{\mathrm{u}}+\mathcal{B}_{\mathrm{cl}, \mathrm{u}} \widehat{z}_{1}, \\
\widehat{z}_{\mathrm{u}}=\mathcal{C}_{\mathrm{cl}, \mathrm{u}} \widehat{x}_{\mathrm{u}}+\mathcal{D}_{\mathrm{cl}, \mathrm{u}} \widehat{z}_{\mathrm{l}},
\end{array}\right. \\
& \mathcal{A}_{\mathrm{cl}, \mathrm{u}}:=\mathcal{A}_{\mathrm{u}}+\mathcal{B}_{\mathrm{u}} \Omega_{\mathrm{u}}\left(I-\mathcal{D}_{\mathrm{u}} \Omega_{\mathrm{u}}\right)^{-1} \mathcal{C}_{\mathrm{u}}, \\
& \mathcal{B}_{\mathrm{cl}, \mathrm{u}}:=\mathcal{B}_{\mathrm{u}}\left(I-\Omega_{\mathrm{u}} \mathcal{D}_{\mathrm{u}}\right)^{-1} \Omega_{\mathrm{ul}},
\end{aligned}
$$

$$
\mathcal{C}_{\mathrm{cl}, \mathrm{u}}:=\left(I-\mathcal{D}_{\mathrm{u}} \Omega_{\mathrm{u}}\right)^{-1} \mathcal{C}_{\mathrm{u}}, \mathcal{D}_{\mathrm{cl}, \mathrm{u}}:=\mathcal{D}_{\mathrm{u}}\left(I-\Omega_{\mathrm{u}} \mathcal{D}_{\mathrm{u}}\right)^{-1} \Omega_{\mathrm{ul}} .
$$

Here, since $\mathcal{G}_{\mathrm{u}} \star \Omega_{\mathrm{u}}$ is admissible and stable as proved, and since $\left(\mathcal{G}_{\mathrm{u}} \star \Omega_{\mathrm{u}}\right) \Omega_{\mathrm{ul}}$ is linear, we can rewrite the condition (28) as a condition with respect to the steady-state gain as in

$$
\left(-\mathcal{C}_{\mathrm{cl}, \mathrm{u}} \mathcal{A}_{\mathrm{cl}, \mathrm{u}}^{-1} \mathcal{B}_{\mathrm{cl}, \mathrm{u}}+\mathcal{D}_{\mathrm{cl}, \mathrm{u}}\right) v_{\mathrm{obj}, \mathrm{l}}=v_{\mathrm{obj}, \mathrm{u}} .
$$

By using $-\mathcal{C}_{\mathrm{u}} \mathcal{A}_{\mathrm{u}}^{-1} \mathcal{B}_{\mathrm{u}}=\Psi_{\mathrm{u}}-\mathcal{D}_{\mathrm{u}}$, we can confirm the term $\mathcal{C}_{\mathrm{cl}, \mathrm{u}} \mathcal{A}_{\mathrm{cl}, \mathrm{u}}^{-1}$ in the above equality reads $\mathcal{C}_{\mathrm{cl}, \mathrm{u}} \mathcal{A}_{\mathrm{cl}, \mathrm{u}}^{-1}=$ $\left(I-\Psi_{\mathrm{u}} \Omega_{\mathrm{u}}\right)^{-1} \mathcal{C}_{\mathrm{u}} \mathcal{A}_{\mathrm{u}}^{-1}$. Therefore we can rewrite (62) as

$$
\left(\left(\Psi_{\mathrm{u}} \Omega_{\mathrm{u}}-I\right)^{-1} \mathcal{C}_{\mathrm{u}} \mathcal{A}_{\mathrm{u}}^{-1} \mathcal{B}_{\mathrm{u}}+\mathcal{D}_{\mathrm{u}}\right)\left(I-\Omega_{\mathrm{u}} \mathcal{D}_{\mathrm{u}}\right)^{-1} \Omega_{\mathrm{ul}} v_{\mathrm{obj}, \mathrm{l}}=v_{\mathrm{obj}, \mathrm{u}}
$$

or equivalently,

$$
\begin{aligned}
& \left(\mathcal{C}_{\mathrm{u}} \mathcal{A}_{\mathrm{u}}^{-1} \mathcal{B}_{\mathrm{u}}-\mathcal{D}_{\mathrm{u}}+\Psi_{\mathrm{u}} \Omega_{\mathrm{u}} \mathcal{D}_{\mathrm{u}}\right)\left(I-\Omega_{\mathrm{u}} \mathcal{D}_{\mathrm{u}}\right)^{-1} \Omega_{\mathrm{ul}} v_{\mathrm{obj}, 1} \\
& =\left(\Psi_{\mathrm{u}} \Omega_{\mathrm{u}}-I\right) v_{\mathrm{obj}, \mathrm{u}} .
\end{aligned}
$$

Again from $-\mathcal{C}_{\mathrm{u}} \mathcal{A}_{\mathrm{u}}^{-1} \mathcal{B}_{\mathrm{u}}+\mathcal{D}_{\mathrm{u}}=\Psi_{\mathrm{u}}$, this holds if and only if $\left(\Psi_{\mathrm{u}} \Omega_{\mathrm{u}}-I\right) v_{\mathrm{obj}, \mathrm{u}}=-\Psi_{\mathrm{u}} \Omega_{\mathrm{ul}} v_{\mathrm{obj}, \mathrm{l}}$. This is equivalent to

$$
\Psi_{\mathrm{u}}\left[\begin{array}{ll}
\Omega_{\mathrm{u}} & \Omega_{\mathrm{ul}}
\end{array}\right]\left[\begin{array}{c}
v_{\mathrm{obj}, \mathrm{u}} \\
v_{\mathrm{obj}, 1}
\end{array}\right]=v_{\mathrm{obj}, \mathrm{u}} .
$$

This completes the proof.

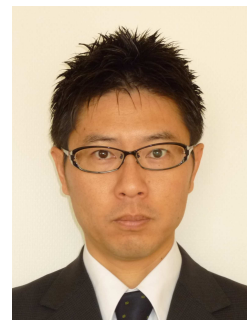

Yoshio Ebihara was born in Fukuoka, Japan, in 1974. He received the B.E., M.E. and D.E. degrees in electrical engineering from Kyoto University, Kyoto, Japan, in 1997, 1999 and 2002, respectively. Since 2002, he has been with the Department of Electrical Engineering, Kyoto University, where he is an Associate Professor since 2010. In 2010, he held a visiting researcher position at LAAS-CNRS Toulouse, France. His research interests include convex optimization in control and positive system analysis and synthesis. Currently, he is an Associate Editor of IEEE Transactions on Control Systems Technology, Automatica, and a board member of IET Control Theory and Applications. He is also a chair of IEEE CSS Technical Committee on Systems with Uncertainty.

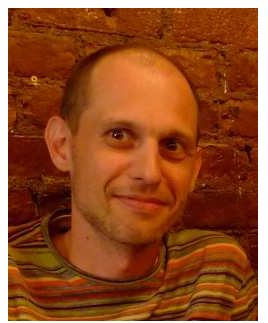

Dimitri Peaucelle was born in Leningrad, USSR, on 2 March 1974. He is a full-time researcher at the French National Center for Scientific Research (CNRS), working at LAAS in Toulouse. He obtained his Ph.D. degree in 2000 from Toulouse University. His research interests are in robust control, and extend to convex optimization over linear matrix inequalities (LMIs), periodic systems, time-delay systems and direct adaptive control. He is also involved in computer-aided control design activities and leads a project for control-oriented software development. He is the main contributor to the Robust Multiobjective Control (RoMulOC) Toolbox. He has been involved in several industrial projects with aerospace partners for launcher, aircraft, and satellite robust control.

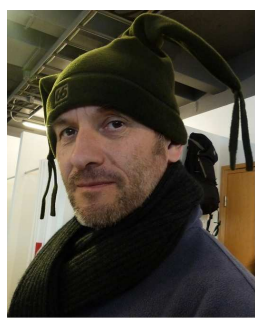

Denis Arzelier was born in Villeneuve Saint Georges, France in 1964. He received the Diplôme d'Ingenieur (Engineer's Degree) from the Ecole Nationale Supérieure des Arts et Métiers, (ENSAM), Paris, 1989, the Diplôme d'Etudes Approfondies (Master's Degree) with specialization in control from the Ecole Centrale, Lille, France, in June 1990. From October 1990 to June 1992 he was a Ph.D. student at the Laboratoire d'Analyse et d'Architecture des Systemes of the Centre National de la Recherche Scientifique (LAAS-CNRS), Toulouse, France. He received the $\mathrm{Ph} . \mathrm{D}$. degree from INSA, Toulouse, France, in June 1992. Since October 1993 he is holding a research position at LAAS-CNRS, Toulouse, France. His research interests are in robust control, convex optimization, and optimal control for aerospace. 